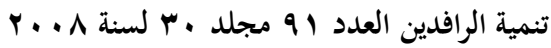

ص ص[rr-r.r.

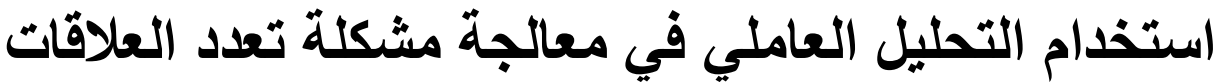 الخطية وتحديد المتغيرات المؤثرة في السعر العالمي للقمح
}

$$
\begin{aligned}
& \text { مزام محمد يميى } \\
& \text { مدرس مساعد } \\
& \text { قسم الإحصاء والمعلوماتية }
\end{aligned}
$$

كلية علوم الحاسبات والرياضيات - جامعة الموصل

\author{
الدكتور ظافر رمضان مطر \\ أستاذ مساعد \\ قسم الإحصاء والمعلوماتية \\ كلية علوم الحاسبات والرياضيات - جامعة الموصل
}

Thafer63@yahoo.com

المستخلص

تعد مشكلة تعدد العلاقات الخطية بين المتغيرات التوضيحية في أنموذج الاتحدار التهار المتعدد من

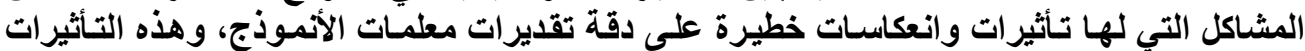

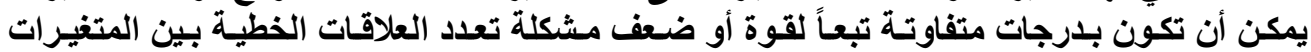

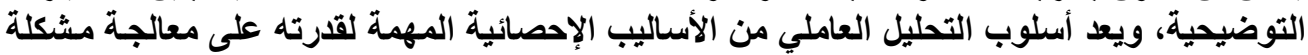

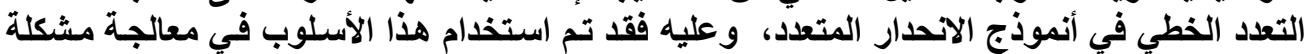

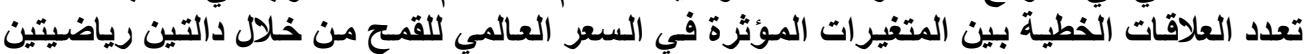

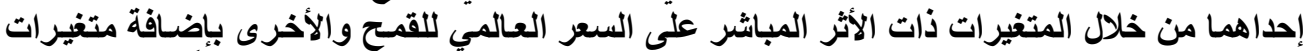

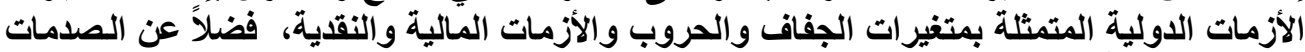

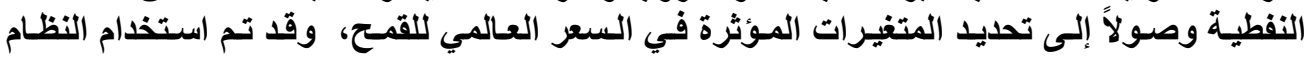
الجاهز MINITAB في الحصول على النتائج.

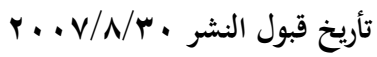

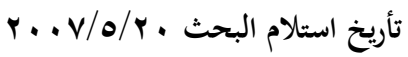




\title{
Using Factor Analysis for Treatment Multicollinearity Problem and to Specifying the Effective Variables on the Wheat World Price
}

Dhafir R. Mutar (PhD)

Assistant Professor

Department of Statistics

University of Mosul

\author{
Mozahem M. Yehya \\ Assistant Lecturer \\ Department of Statistics \\ University of Mosul
}

\begin{abstract}
The Multicollinearity among the explanatory variables in the Multiple Regression Model is a problem that has effects and serious reflections on the accuracy estimation of the Model Parameters, and these effects may be varied in degrees according to the strength or weakness of the multicollinearity among the explanatory variables. The factors analysis is one of the important statistics manners that capable to the treatment of the multicollinearity in the Multiple Regression Model. This manner is used to Treat the Multicollinearity problem and to specifying the effective variables on the wheat world prices by two mathematical functions. The first one is by the variables that direct the effects to the wheat world prices and the other by adding the crises internationalism variables which is curtness, wars, finance and monetary crises and petrol shocks in order to estimate the parameters used (MINITAB) Package.
\end{abstract}

المقدمة

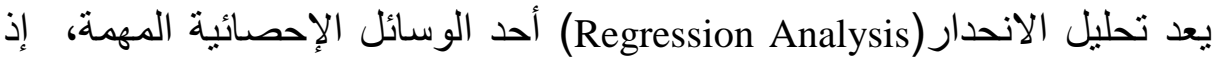

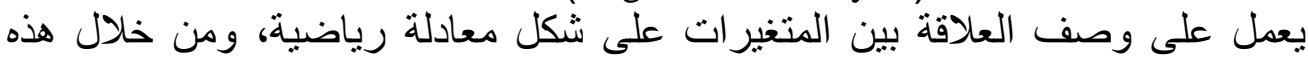

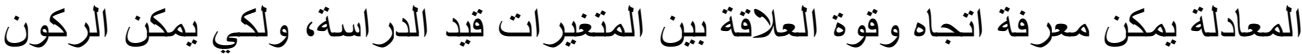

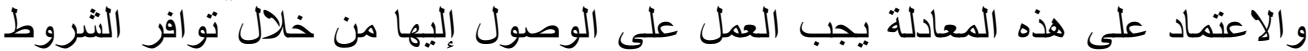

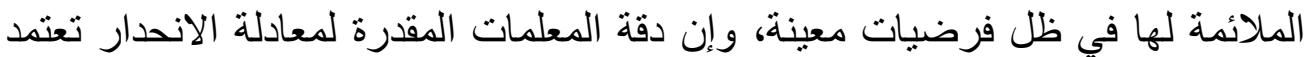
بشكل أساس على صحة هذه الفرضيات. إن تحليل الانحدار يستخدم بوصفية أداة تحلية النيلية لعدة أسباب، منها الحاجة المبكرة لتطوير التقدير بوصفه علاقة دالية بمكن استخدامها

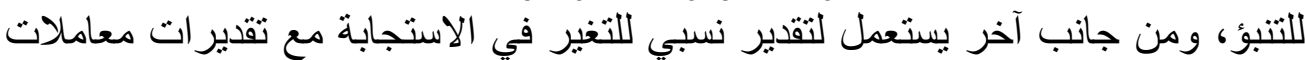
الانحدار، وقد تتشأ المشاكل بسبب أن البيانات المتوفرة لا يمكن بيساطة أن تدعم الفكرة

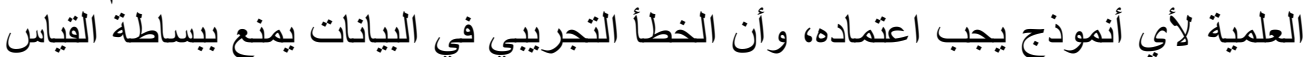

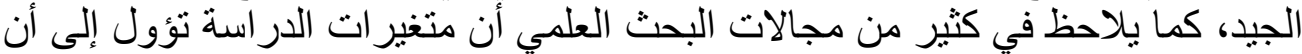

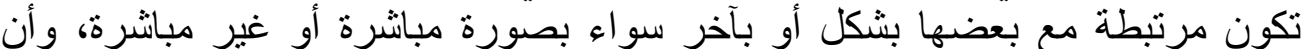

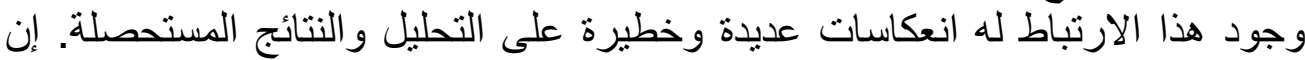
العلاقات الخطية المتعددة (Multicollinearity) تظهر عندما يكون هناك متغير توضيحي (Correlated) مع متغير توضيحي آخر أو مجمو عة تونة (Explanatory Variable) من المتغير ات التوضيحية ذات اتجاه خطي وهذه العلاقات الخطية المتّاخلة من العلاقات

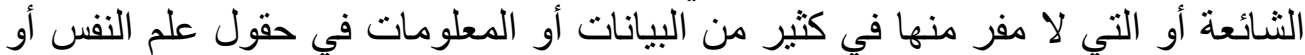




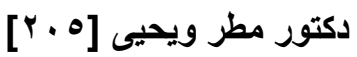

الاقتصاد أو إدارة الأعمال أو الجغرافية، و هذا صحيح لأنه يصعب في كثير من الأحيان

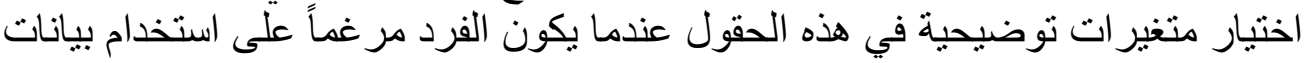

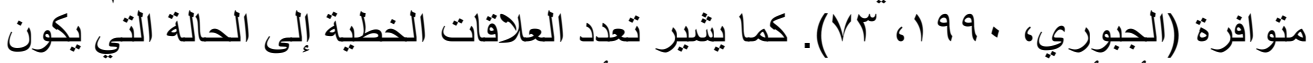

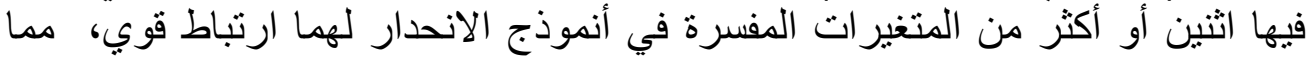

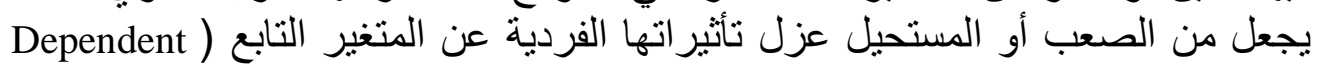

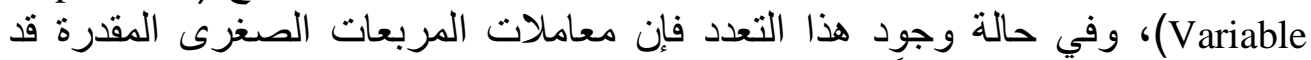

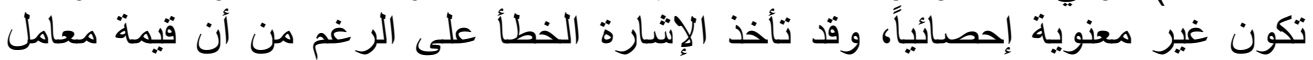

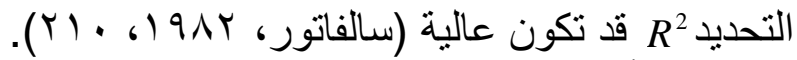

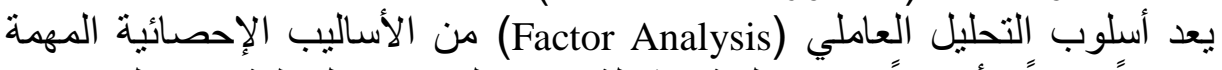

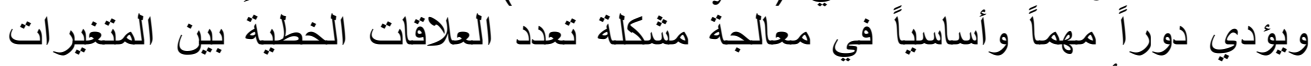
التوضيحية لأنموذج الانحدار المتعدد.

يهذف البحث إلى تحديد المتغيرات التي لها دور معنوي في التأثير على السعر هدف البحث

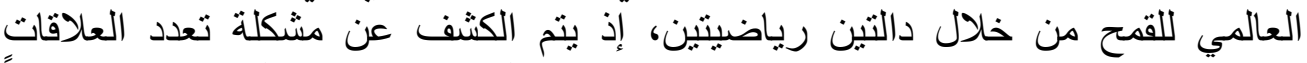

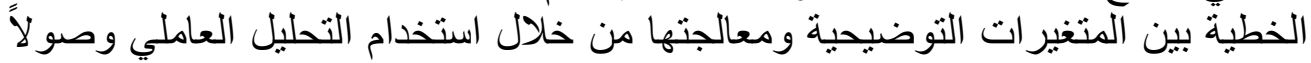
إلى تقدير معادلة انحدارية للسعر العالمي للقمح تتصف بخصنيائص إحصائية جيدة.

إن المؤشرات المهمة الدالة على مشكلة تعدد العلاقات الخطية في أنموذج الانحدار

$$
\text { الجاتب النظري }
$$

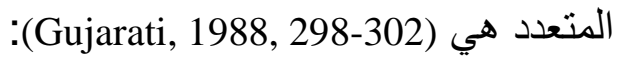

ا. معنوية الارتباطات بين كل زوج من من المتنغيرات التوضيحية في الأنموذج، وهذه

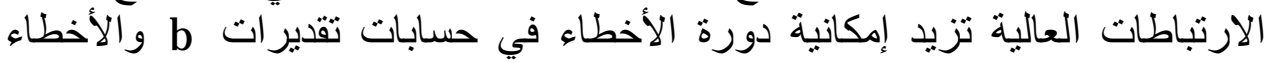

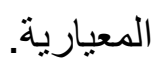

r. عند وجود أكثر من متغيرين توضيحيين (X) مثناً، ربما يكون لها ارتباط

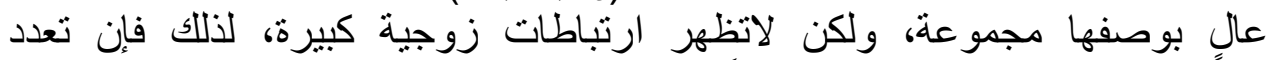

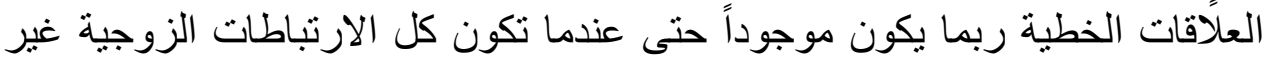

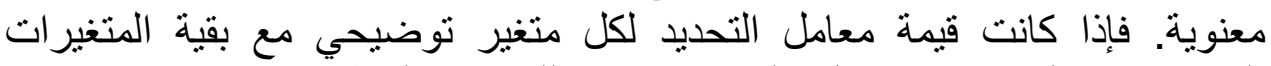

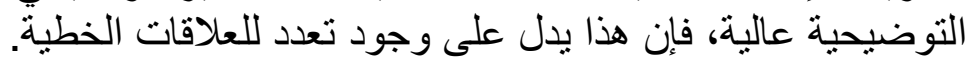

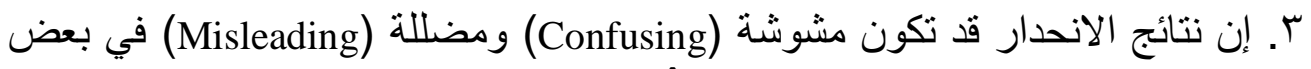

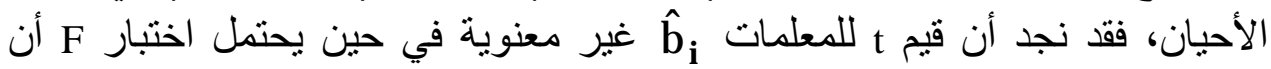

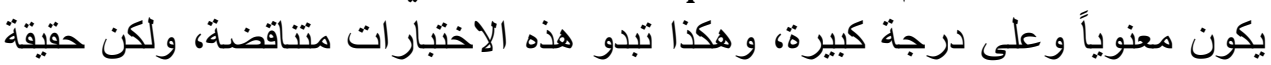
هي ليست كذلك، إذ إن اختبار t يشير إلى إسهام متغير واحد فقط، في حين أن معنوية 
اختبار F تعطي دلالة على أن هناك على الأقل متغير واحد من المتغيرين يقدم إسهاماً

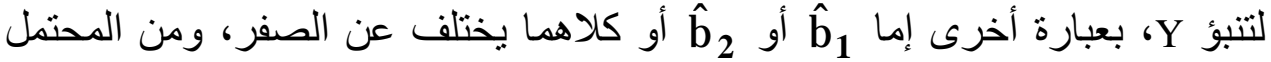

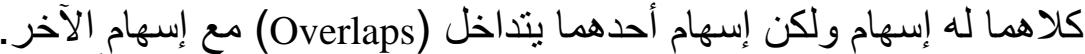

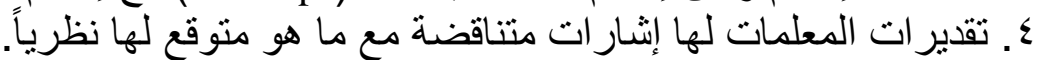

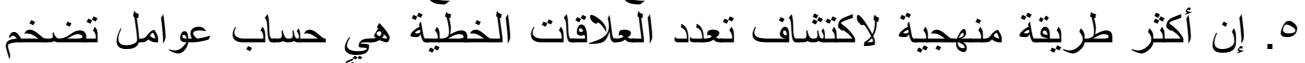

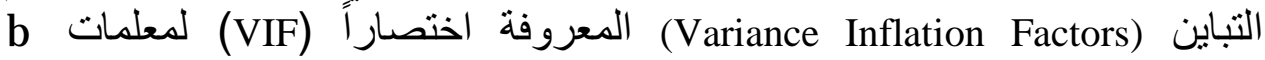

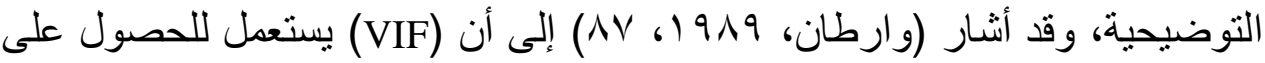

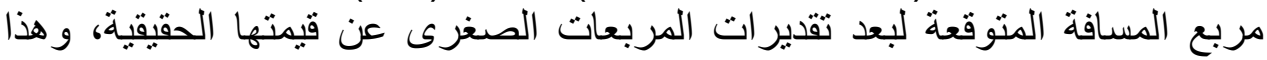

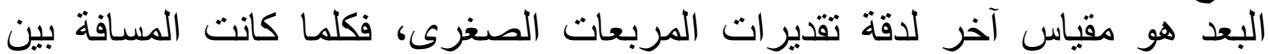

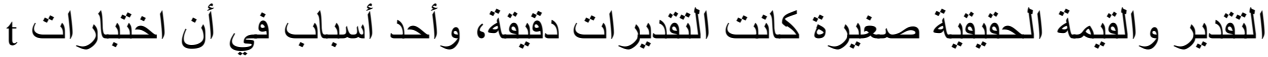
S $_{\hat{\mathbf{B}}_{\mathbf{i}}}$ لمعلمات التي تتضخم عند وجود تعدد للعلاقات الخطية. وفي الانحدار المتعدد من المكن

$$
S_{\hat{\mathbf{B}}_{i}}^{2}=S^{2}\left(\frac{1}{1-\mathbf{R}_{\mathrm{i}}^{2}}\right)
$$

إذ إن Sو $\mathbf{S}^{2}$

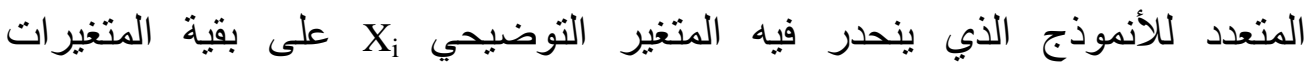
التوضيحية، وأن الكمية

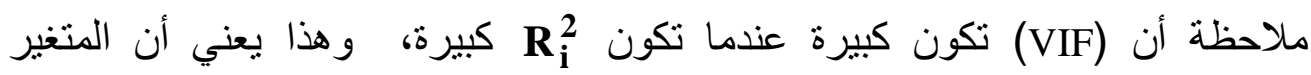

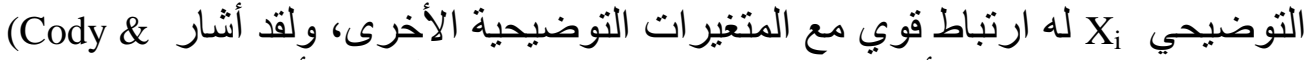

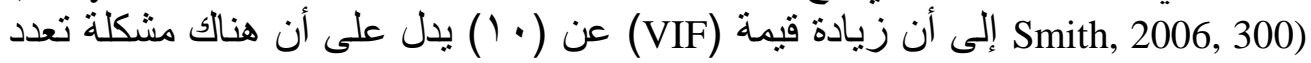

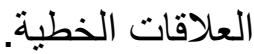

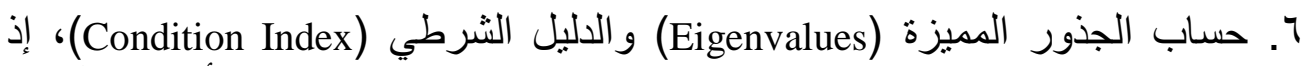

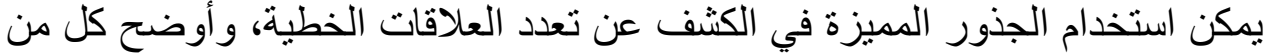

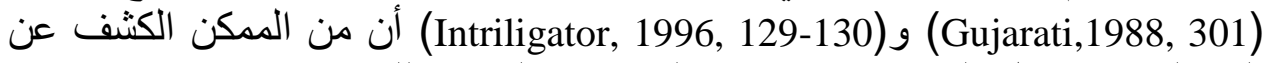

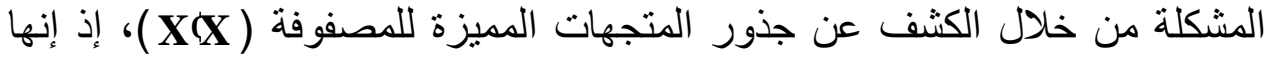

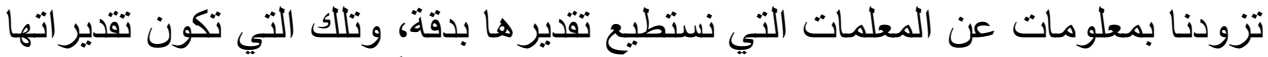

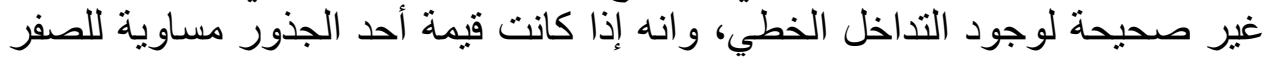

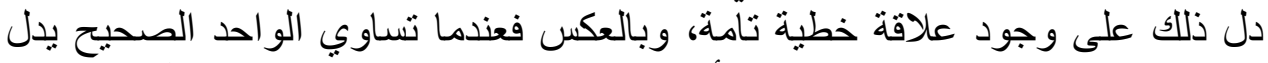

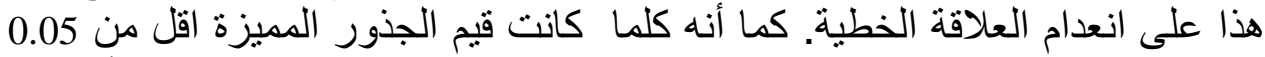
فهذا يعني وجود معادلات تربط بين المتغير اتهات التوضيحية بعدد هذه الجذور. أما فيما 
[Y.V. [كتور مطر ويحيى

يخص الاليل الثرطي والذي هو عبارة عن حاصل قسمة أكبر جذر مميز على كل الذبل الجذور المميزة فيأخذ الصيغة الآيتة:

$$
\mathrm{C}_{\mathrm{i}}=\frac{\lambda_{\max }}{\lambda_{\mathrm{i}}} \quad \mathrm{i}=1,2, \ldots, \mathrm{k}
$$

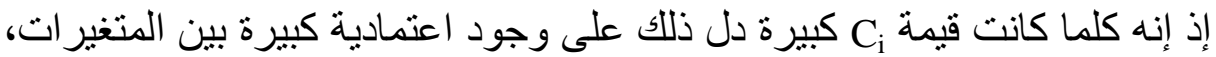

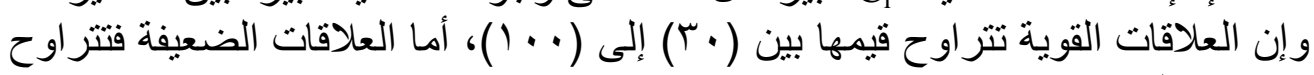
بين (0) إلى ( • (1) (Intriligator, 1996, 129). V. القيمة المرتفعة لمعامل التحديد ( تقدم مؤشر اً آخر على وجود مشكلة التعدد الخطي.

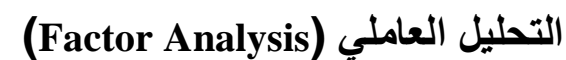

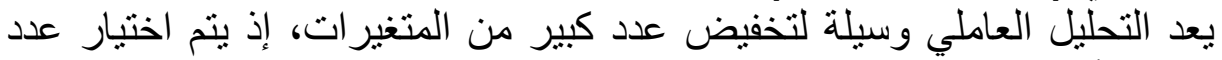

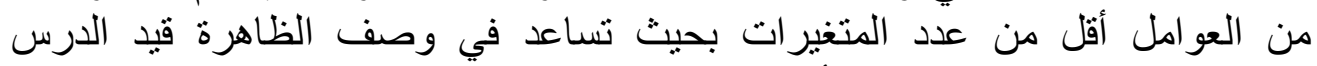

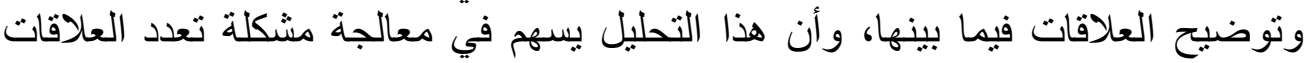

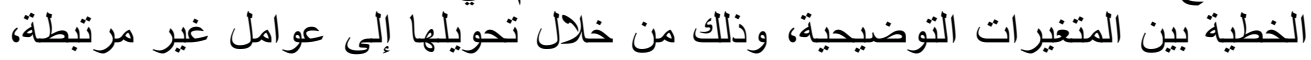

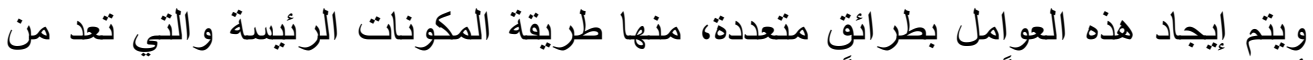
أكثر الطر ائق شيو عاً واستخداماً (Brown, 2006, 12).

طريقة المكونات الرئيسة (Principal Components Method) تعمل هذه الطريقة على المئى تحويل المتغيرات التوضيحية المرتبطة خطياً (إلى تر اكيب خطية متعامدة $\left(\mathbf{X}_{\mathbf{1}}, \mathbf{X}_{\mathbf{2}}, \ldots, \mathbf{X}_{\mathbf{K}}\right)$

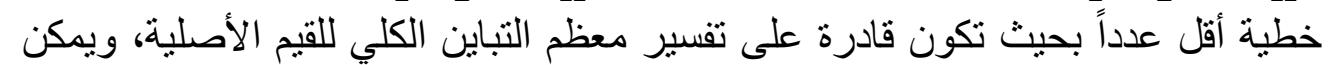

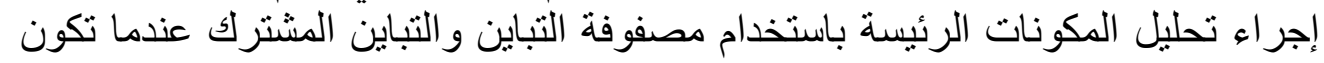

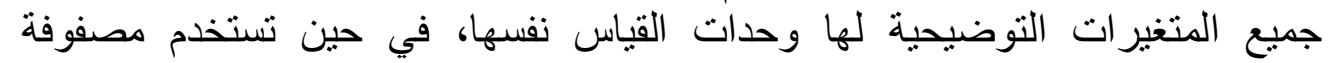

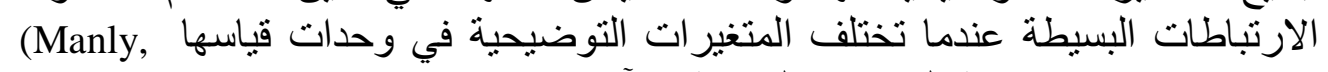
2004, 94) $P_{J}=a_{1 j} X_{1}+a_{2 j} X_{2}+\ldots+a_{k j} \quad \ldots j=1,2, \ldots, k$

$$
\begin{aligned}
& \text { ويمكن كتابتها بصيغة المصفوفات وبالثكل الآتي: P } \\
& \text { : Pصفوفة المكونات الرئيسة ( }) \text { P }
\end{aligned}
$$

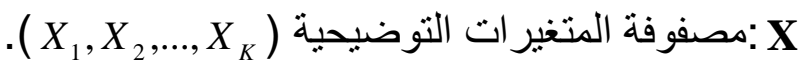

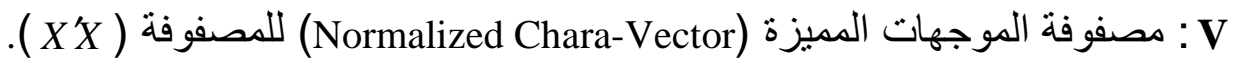


تتصف المكونات الرئيسة بكونها متعامدة (غبر مرتبطة)، كما أن المكون الرئيس

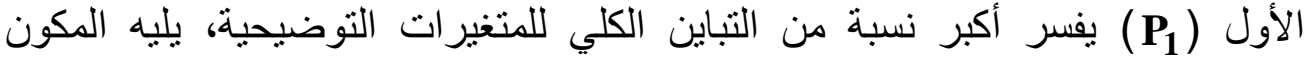

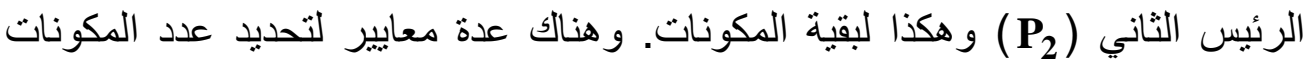

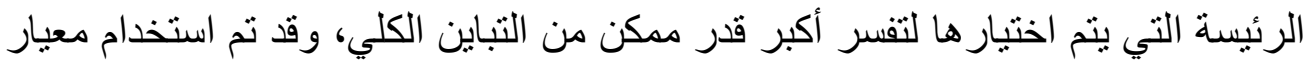
الذي يستند على رسم الجذور المميزة (

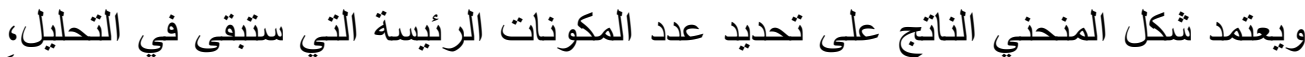

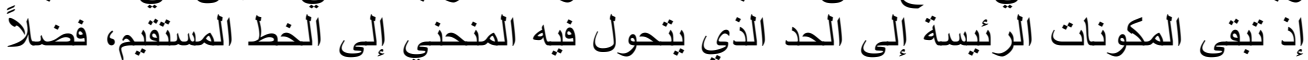
عن معيار اختيار المكونات التي يكون تباينها أكبر من واحد ولاسيما لالئي بعد التدوير.

تدوير العوامل (Factor Rotation)

إن الهدف من عملية التدوير هو الحصول على أفضل التحميلات وتعزيز قابلية

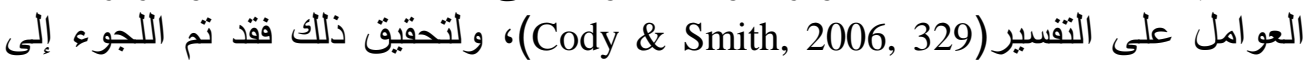
التدوير باستخدام طريقة تعظيم التباين (Varimax)، أي التنوير المتعامد.

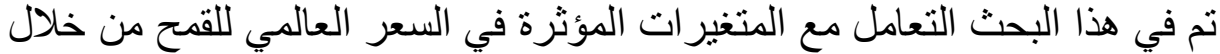

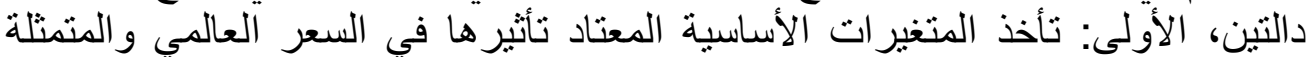

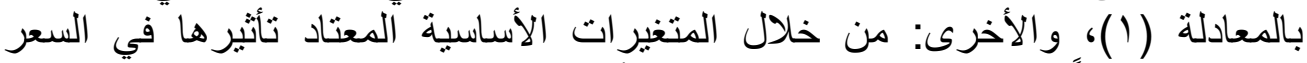

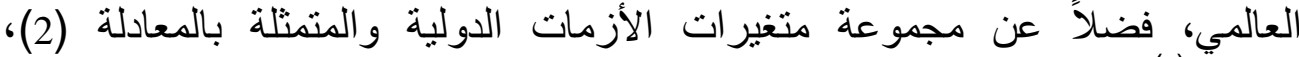

$$
\begin{aligned}
& Y=f\left(X_{1}, X_{2}, X_{3}, X_{4}, X_{5}, X_{6}, X_{7}, X_{8}, X_{9}, X_{10}\right) \\
& Y=f\left(X_{1}, X_{2}, X_{3}, X_{4}, X_{5}, X_{6}, X_{7}, X_{8}, X_{9}, X_{10}, X_{11}, X_{12}, X_{13}, X_{14}\right)
\end{aligned}
$$

Y X X X

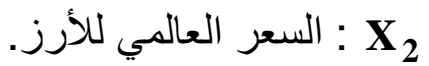

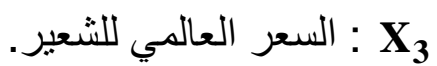

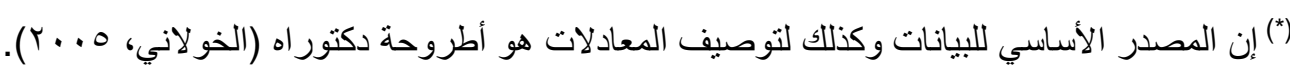

$$
\begin{aligned}
& \text { أن } 11 \text { :عامل الحروب. }
\end{aligned}
$$

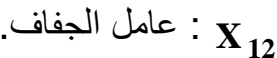




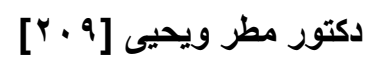

$$
\begin{aligned}
& \text { X X } \\
& \text { X5 } \\
& \text { X X } \\
& \text { X } \\
& \text { X8 } \\
& \text { : X } \\
& \text { : } \mathbf{X}_{10}
\end{aligned}
$$

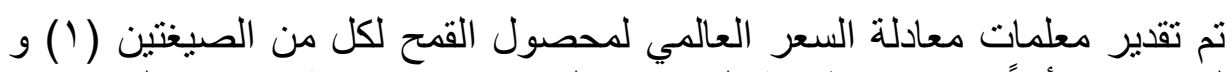
(Y) المذكورتين أنفاً باستخدام طريقة المربعات الصغرى الصغ الاعتيادية، وكانت النتائج كما

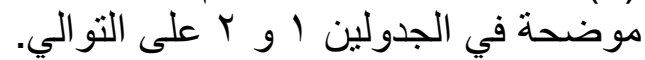
يتضح من الجدول / معنوية كل من المتغيرات

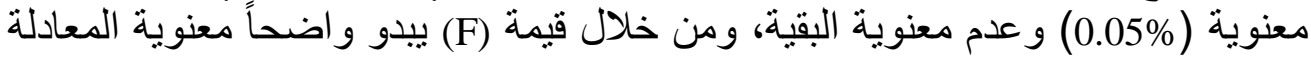

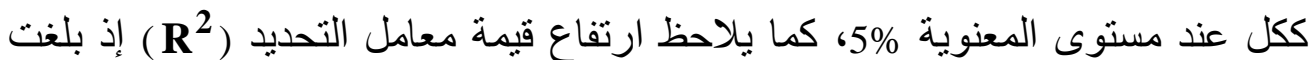

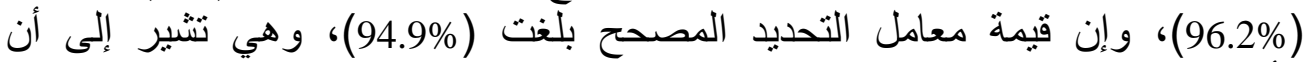

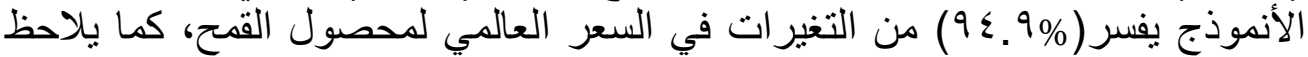
من الجدول ب معنوية المتغيرين (P)

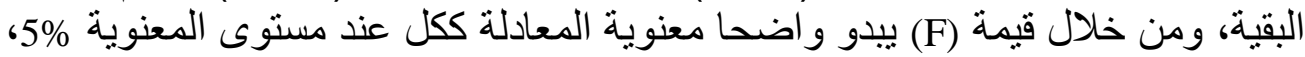

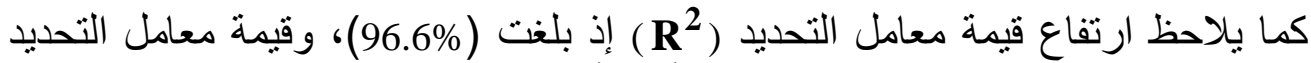
المصحح بلغت (94.9\%)، وهي تثير إلى أن الأنموذج يفسر (194.9\%) من النغير (النغيرات في السعر العالمي لمحصول القمح.

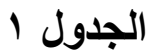

تقدير معادلة السعر العالمي للقمح باستبعاد متغيرات الأزمات الدولية مع نتائج التحليل الإحصائي وتحليل التبائين 


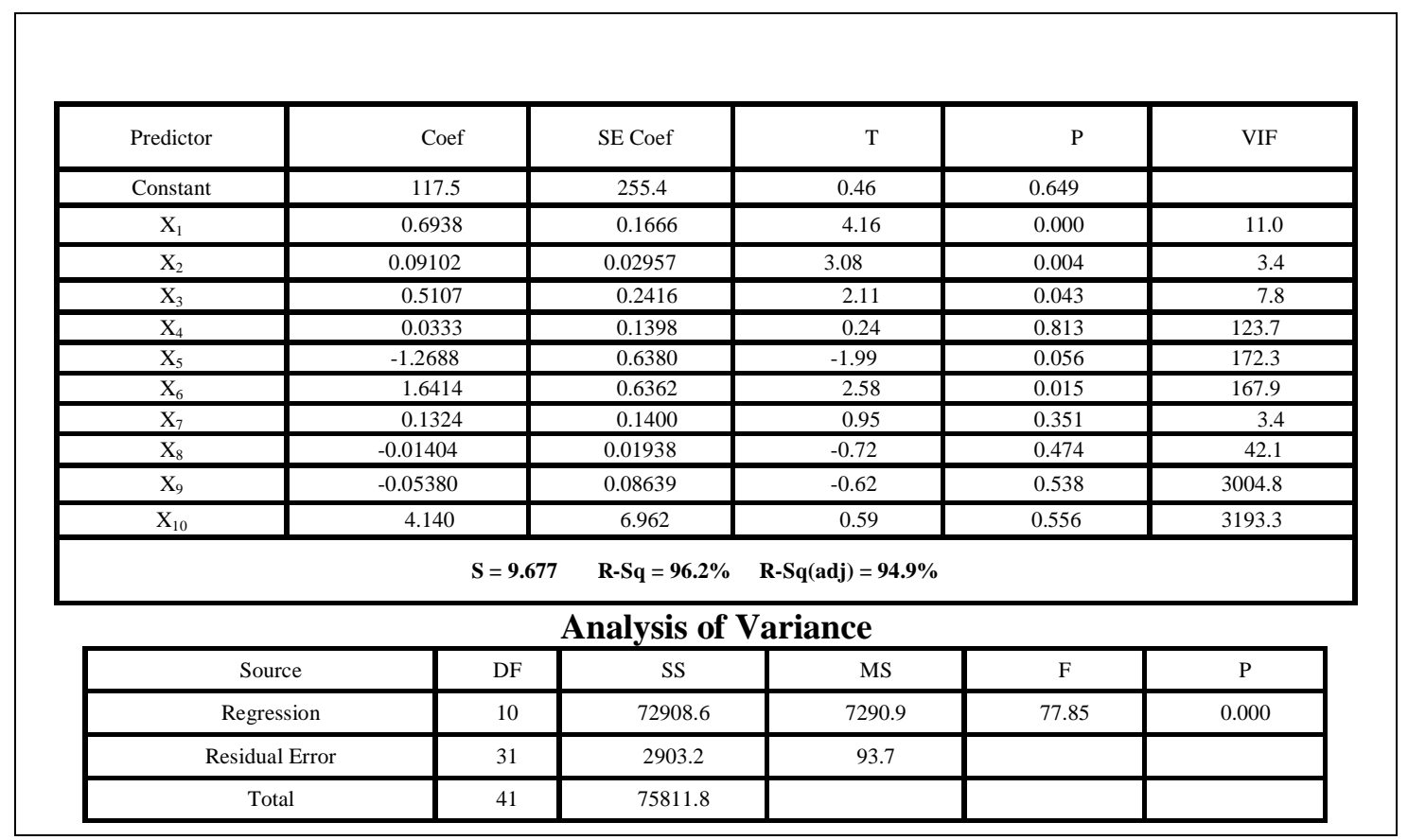

\section{الكثف عن مشكلة تعدد العلاقات الخطية}

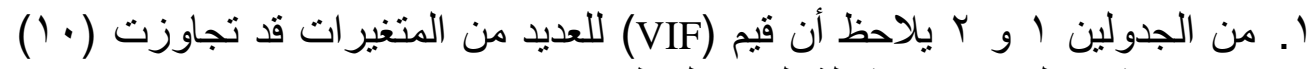
و هذا مؤشر على وجود مشكلة التعدد الخطي.

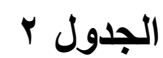

تقدير معادلة السعر العالمي للقمح فضلا عن مجموعة بالئ متغيرات الأزمات الدولية مع نتائج التحليل الإحصائي وتحليل التباين 
دكتور مطر ويحيى [Yl']

\begin{tabular}{|c|c|c|c|c|c|}
\hline Predictor & Coef & SE Coef & $T$ & $P$ & VIF \\
\hline Constant & -461.1 & 414.7 & -1.11 & 0.276 & 12.3 \\
\hline $\mathrm{X} 1$ & 0.7381 & 0.1764 & 4.18 & 0.000 & 4.3 \\
\hline $\mathrm{X} 2$ & 0.06833 & 0.03365 & 2.03 & 0.052 & 8.8 \\
\hline $\mathrm{X} 3$ & 0.4453 & 0.2571 & 1.73 & 0.095 & 180.1 \\
\hline $\mathrm{X} 4$ & 0.1326 & 0.1694 & 0.78 & 0.441 & 184.9 \\
\hline $\mathrm{X} 5$ & -1.2192 & 0.6638 & -1.84 & 0.077 & 190.0 \\
\hline $\mathrm{X} 6$ & 1.5264 & 0.6797 & 2.25 & 0.033 & 5.3 \\
\hline $\mathrm{X} 7$ & 0.1933 & 0.1755 & 1.10 & 0.280 & 53.6 \\
\hline $\mathrm{X} 9$ & -0.02649 & 0.02195 & -1.21 & 0.238 & 8471.6 \\
\hline $\mathrm{X} 10$ & 0.1357 & 0.1368 & 0.99 & 0.330 & 1.5 \\
\hline $\mathrm{X} 11$ & -10.96 & 11.21 & -0.98 & 0.337 & 1.7 \\
\hline $\mathrm{X} 12$ & -0.223 & 3.735 & -0.06 & 0.953 & 1.9 \\
\hline $\mathrm{X} 14$ & 2.507 & 3.990 & 0.63 & 0.535 & 5.0 \\
\hline
\end{tabular}

Analysis of Variance

\begin{tabular}{|c|c|c|c|c|c|}
\hline Source & DF & SS & MS & F & P \\
\hline Regression & 14 & 73261.0 & 5232.9 & 55.39 & 0.000 \\
\hline Residual Error & 27 & 2550.8 & 94.5 & & \\
\hline Total & 41 & 75811.8 & & & \\
\hline
\end{tabular}

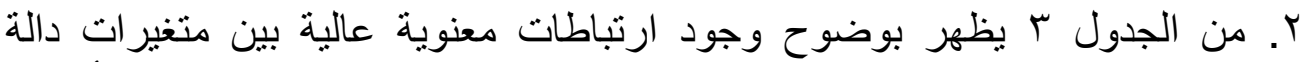

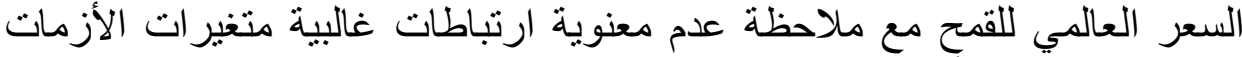

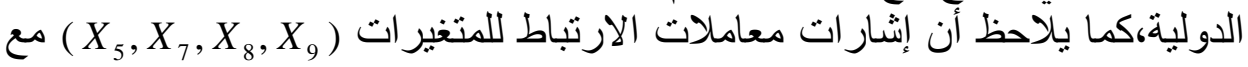

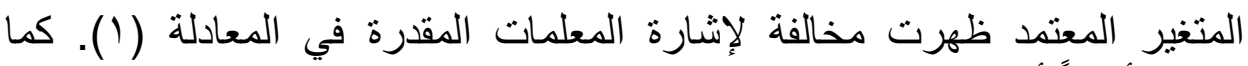
يلاحظ أيضاً أن إثار ات معاملات الارنباط للمتغيرات

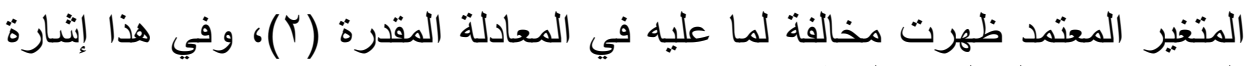
إلى وجود مشكلة التعدد الخطي.

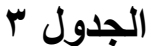

مصفوفة معاملات الارتباط البسيط بين المتغيرات 


\begin{tabular}{|c|c|c|c|c|c|c|c|c|c|c|c|c|c|c|}
\hline & $\mathrm{Y}$ & $x_{1}$ & $\mathrm{X}_{1}$ & $x_{3}$ & $x_{4}$ & $\mathbf{X}$, & $\mathrm{X}_{s}$ & $\mathrm{x}$, & $x_{4}$ & $X_{s}$ & $\mathbf{X}_{20}$ & $x_{n}$ & $x_{11}$ & $\mathbf{x}_{\mathrm{D}}$ \\
\hline$x_{4}$ & $\begin{array}{l}0.96 \\
0.00\end{array}$ & & & & & & & & & & & & & \\
\hline$x_{1}$ & $\begin{array}{l}0.788 \\
0.000 \\
\end{array}$ & $\begin{array}{l}.775 \\
0.000 \\
\end{array}$ & & & & & & & & & & & & \\
\hline $\mathbf{x}_{\boldsymbol{j}}$ & $\begin{array}{r}.749 \\
6.000 \\
\end{array}$ & $\begin{array}{l}0.714 \\
0.000 \\
\end{array}$ & $\begin{array}{l}.491 \\
0.001 \\
\end{array}$ & & & & & & & & & & & \\
\hline$x_{4}$ & $\begin{array}{l}.703 \\
0.000 \\
\end{array}$ & $\begin{array}{l}.670 \\
0.0700 \\
\end{array}$ & $\begin{array}{l}0.330 \\
0.0133 \\
0\end{array}$ & $\begin{array}{l}0.622 \\
0.000 \\
\end{array}$ & & & & & & & & & & \\
\hline $\mathrm{X}_{s}$ & $\begin{array}{l}0.699 \\
0.000 \\
\end{array}$ & $\begin{array}{l}.652 \\
0.000 \\
\end{array}$ & $\begin{array}{l}0.311 \\
0.045 \\
\end{array}$ & $\begin{array}{l}0.646 \\
0.000\end{array}$ & $\begin{array}{l}0.961 \\
0.060 \\
\end{array}$ & & & & & & & & & \\
\hline$x_{*}$ & $\begin{array}{l}.716 \\
2.000 \\
\end{array}$ & $\begin{array}{l}0.663 \\
0.000 \\
\end{array}$ & $\begin{array}{l}.3230 \\
0.039 \\
\end{array}$ & $\begin{array}{l}0.657 \\
0.000 \\
\end{array}$ & $\begin{array}{l}.962 \\
0.060 \\
\end{array}$ & $\begin{array}{l}0.996 \\
0.0960 \\
.000\end{array}$ & & & & & & & & \\
\hline $\mathbf{x}_{\tau}$ & $\begin{array}{r}0.119 \\
0.454 \\
\end{array}$ & $\begin{array}{r}-0.136 \\
0.325 \\
\end{array}$ & $\begin{array}{r}-0.122 \\
0.441 \\
\end{array}$ & $\begin{array}{r}-0.150 \\
0.344 \\
\end{array}$ & $\begin{array}{r}-6.122 \\
0.443\end{array}$ & $\begin{array}{r}0.603 \\
0.983\end{array}$ & $\begin{array}{r}-0.023 \\
0.854\end{array}$ & & & & & & & \\
\hline$x_{*}$ & $\begin{array}{l}0.462 \\
0.002 \\
\end{array}$ & $\begin{array}{l}.399 \\
0.009\end{array}$ & $\begin{array}{l}0.690 \\
0.572\end{array}$ & $\begin{array}{l}0.696 \\
0.000\end{array}$ & $\begin{array}{l}.834 \\
0.000\end{array}$ & $\begin{array}{l}.858 \\
2.000\end{array}$ & $\begin{array}{l}0.855 \\
0.060\end{array}$ & $\begin{array}{l}0.028 \\
0.562\end{array}$ & & & & & & \\
\hline$X_{s}$ & $\begin{array}{l}.653 \\
0.000 \\
\end{array}$ & $\begin{array}{l}.6022 \\
0.000 \\
\end{array}$ & $\begin{array}{l}6.272 \\
0.0822 \\
\end{array}$ & $\begin{array}{l}0.669 \\
0.000 \\
0\end{array}$ & $\begin{array}{l}.9 .971 \\
0.000 \\
\end{array}$ & $\begin{array}{l}.964 \\
0.0000 \\
\end{array}$ & $\begin{array}{l}0.944 \\
0.000 \\
\end{array}$ & $\begin{array}{l}0.011 \\
0.445 \\
\end{array}$ & $\begin{array}{l}0.926 \\
0.0000 \\
\end{array}$ & & & & & \\
\hline $\mathbf{x}_{\infty}$ & $\begin{array}{l}0.664 \\
0.000\end{array}$ & $\begin{array}{l}8.615 \\
0.000\end{array}$ & $\begin{array}{l}0.285 \\
0.067 \\
\end{array}$ & $\begin{array}{l}0.665 \\
0.000\end{array}$ & $\begin{array}{l}.9773 \\
0.000 \\
\end{array}$ & $\begin{array}{l}0.966 \\
0.900\end{array}$ & $\begin{array}{l}0.966 \\
0.000\end{array}$ & $\begin{array}{l}0.699 \\
0.954\end{array}$ & $\begin{array}{l}0.918 \\
0.000\end{array}$ & $\begin{array}{l}1.00000 \\
0.000\end{array}$ & & & & \\
\hline $\mathrm{X}_{\mathrm{a}}$ & $\begin{array}{r}-0.076 \\
2.633 \\
\end{array}$ & $\begin{array}{r}-0.116 \\
0.4663 \\
\end{array}$ & $\begin{array}{r}.008 \\
0.958 \\
\end{array}$ & $\begin{array}{r}-0.247 \\
0.114 \\
\end{array}$ & $\begin{array}{l}0.106 \\
0.505 \\
\end{array}$ & $\begin{array}{l}0.102 \\
0.519 \\
\end{array}$ & $\begin{array}{l}0.095 \\
0.551 \\
\end{array}$ & $\begin{array}{l}0.033 \\
0.836 \\
\end{array}$ & $\begin{array}{l}0.005 \\
0.976 \\
0.97\end{array}$ & $\begin{array}{l}0.0788 \\
0.622 \\
\end{array}$ & $\begin{array}{l}0.0799 \\
0.617 \\
\end{array}$ & & & \\
\hline $\mathrm{X}_{12}$ & $\begin{array}{l}0.609 \\
0.953 \\
\end{array}$ & $\begin{array}{r}-0.624 \\
0.8881 \\
\end{array}$ & $\begin{array}{r}-0.007 \\
0.963 \\
\end{array}$ & $\begin{array}{r}-0.129 \\
0.415 \\
\end{array}$ & $\begin{array}{r}-0.075 \\
0.637 \\
\end{array}$ & $\begin{array}{l}.003 \\
0.987 \\
\end{array}$ & $\begin{array}{r}-0.005 \\
0.976 \\
\end{array}$ & $\begin{array}{r}0.359 \\
0.011 \\
\end{array}$ & $\begin{array}{l}-0.1117 \\
0.500 \\
\end{array}$ & $\begin{array}{l}-0.0771 \\
0.6557 \\
\end{array}$ & $\begin{array}{r}-0.062 \\
0.096 \\
\end{array}$ & $\begin{array}{r}-0.168 \\
0.287 \\
\end{array}$ & & \\
\hline$x_{0}$ & $\begin{array}{l}.142 \\
0.371\end{array}$ & $\begin{array}{l}0.182 \\
0.250\end{array}$ & $\begin{array}{l}0.118 \\
0.456 \\
\end{array}$ & $\begin{array}{l}0.079 \\
0.617\end{array}$ & $\begin{array}{l}0.230 \\
0.144 \\
\end{array}$ & $\begin{array}{l}0.169 \\
0.286\end{array}$ & 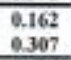 & $\begin{array}{r}-0.048 \\
0.765 \\
\end{array}$ & $\begin{array}{l}0.054 \\
0.736\end{array}$ & $\begin{array}{l}0.193 \\
0.2220 \\
\end{array}$ & $\begin{array}{l}0.194 \\
0.217 \\
\end{array}$ & $\begin{array}{r}-10.028 \\
0.0661 \\
\end{array}$ & $\begin{array}{l}-0.028 \\
0.860\end{array}$ & \\
\hline $\mathbf{X}_{\mathrm{iv}}$ & $\begin{array}{l}0.372 \\
0.015\end{array}$ & $\begin{array}{l}0.331 \\
0.032\end{array}$ & $\begin{array}{l}8.497 \\
0.000 \\
\end{array}$ & $\begin{array}{l}0.259 \\
0.064\end{array}$ & $\begin{array}{l}8.044 \\
0.780 \\
\end{array}$ & $\begin{array}{l}0.072 \\
0.652 \\
\end{array}$ & $\begin{array}{l}0.077 \\
0.628\end{array}$ & $\begin{array}{r}-0.132 \\
0.404 \\
\end{array}$ & $\begin{array}{l}0.097 \\
0.541\end{array}$ & $\begin{array}{l}0.065 \\
0.651\end{array}$ & $\begin{array}{l}0.079 \\
0.621\end{array}$ & $\begin{array}{c}-0.059 \\
0.708\end{array}$ & $\begin{array}{l}0.027 \\
0.863\end{array}$ & $\begin{array}{r}-0.357 \\
0.0220\end{array}$ \\
\hline
\end{tabular}

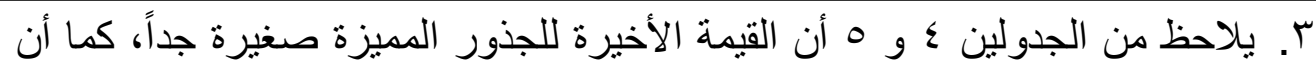

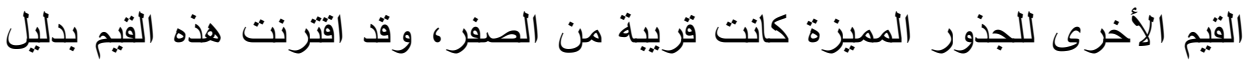

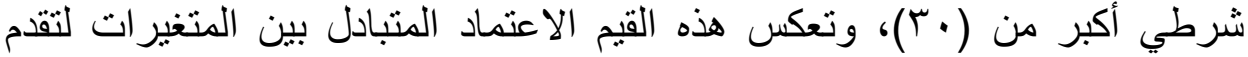

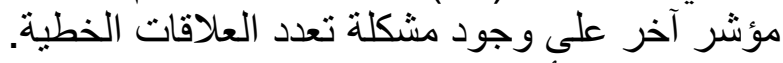
ع. لغرض تحديد أي من المتغير ات التوضيحية المسببة لمشكلة التعدد الخطي للعلاقة التهاتة

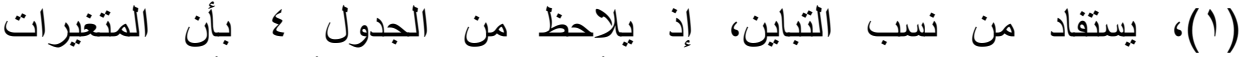
لها نسب تباين أكبر من ه. • هـ كما أن لها أقل قيم للجذور

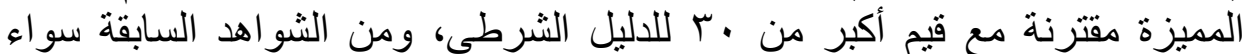

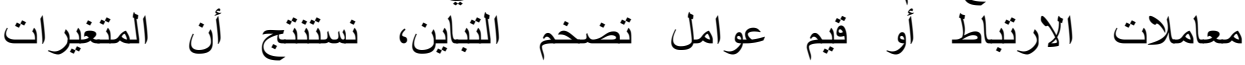
هي المسببة لمشكلة التعدد الخطي. 
دكتور مطر ويحيى [Y I I

الجذور المميزة والدليل الشرطي ونسب تحلل التباين لمتغيرات السعر العالمي للقمح

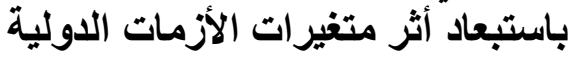

\begin{tabular}{|c|c|c|c|c|c|c|c|c|c|c|c|c|}
\hline Numbe & Eigenvalue & $\begin{array}{c}\text { Condition } \\
\text { Index }\end{array}$ & $\begin{array}{c}\text { Var } \\
\text { Prop } \\
\text { X1 }\end{array}$ & $\begin{array}{c}\text { Var } \\
\text { Prop } \\
\text { X2 }\end{array}$ & $\begin{array}{c}\text { Var } \\
\text { Prop } \\
\text { X3 }\end{array}$ & $\begin{array}{c}\text { Var } \\
\text { Prop } \\
\text { X4 }\end{array}$ & $\begin{array}{c}\text { Var } \\
\text { Prop } \\
\text { X5 }\end{array}$ & $\begin{array}{c}\text { Var } \\
\text { Prop } \\
\text { X6 }\end{array}$ & $\begin{array}{c}\text { Var } \\
\text { Prop } \\
\text { X7 }\end{array}$ & $\begin{array}{c}\text { Var } \\
\text { Prop } \\
\text { X8 }\end{array}$ & $\begin{array}{c}\text { Var } \\
\text { Prop } \\
\text { X9 }\end{array}$ & $\begin{array}{c}\text { Var } \\
\text { Prop } \\
\text { X10 }\end{array}$ \\
\hline 1 & 9.46002 & 1.00000 & 0.0001 & 0.0003 & 0.0001 & 0.0000 & 0.0000 & 0.0000 & 0.0002 & 0.0001 & 0.0000 & 0.0000 \\
\hline 2 & 0.96148 & 3.13672 & 0.0000 & 0.0000 & 0.0000 & 0.0000 & 0.0000 & 0.0000 & 0.2843 & 0.0002 & 0.0000 & 0.0000 \\
\hline 3 & 0.42268 & 4.73089 & 0.0009 & 0.0118 & 0.0003 & 0.0000 & 0.0000 & 0.0000 & 0.0129 & 0.0141 & 0.0000 & 0.0000 \\
\hline 4 & 0.08229 & 10.72169 & 0.0135 & 0.1678 & 0.0003 & 0.0002 & 0.0001 & 0.0000 & 0.0026 & 0.0008 & 0.0000 & 0.0000 \\
\hline 5 & 0.04527 & 14.45527 & 0.0013 & 0.0202 & 0.0559 & 0.0008 & $0.0011 \cdot$ &. .0011 & 0.0005 & 0.0503 & 0.0000 & 0.0002 \\
\hline 6 & 0.01761 & 23.17756 & 0.1863 & 0.6330 & 0.0690 & 0.0002 & 0.0001 & 0.0002 & 0.0075 & 0.0125 & 0.0000 & 0.0000 \\
\hline 7 & 0.00647 & 38.23471 & 0.1124 & 0.0632 & 0.0303 & 0.0073 & 0.0245 & 0.0266 & 0.0024 & 0.0007 & 0.0001 & 0.0014 \\
\hline 8 & 0.00342 & 52.56580 & 0.5386 & 0.0033 & 0.6673 & 0.0069 & $0.0012 . \cdot$ & 0.006 & 0.0075 & 0.3961 & 0.0000 & 0.0041 \\
\hline 9 & 0.0005033 & 137.10271 & 0.0002 & 0.0242 & 0.0038 & 0.4580 & 0.1838 & 0.2196 & 0.1963 & 0.2192 & 0.0018 & 0.0180 \\
\hline 10 & 0.0002434 & 197.15298 & 0.0018 & 0.0502 & 0.0025 & 0.5236 & 0.7766 & 0.7394 & 0.4859 & 0.3042 & 0.0015 & 0.0231 \\
\hline 11 & $8.63606 \mathrm{E}-6$ & 1047 & 0.1448 & 0.0257 & 0.1705 & 0.0030 & 0.0128 & 0.0125 & 0.0000 & 0.0018 & 0.9966 & 0.9531 \\
\hline
\end{tabular}

๑. من الجدول • ولغرض تحديد أي من المتغير ات التوضيحية المسببة لمشكلة التعدد

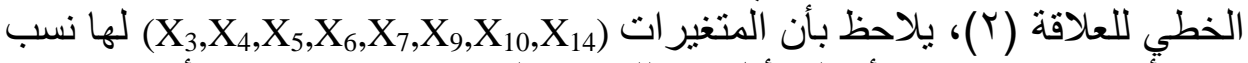

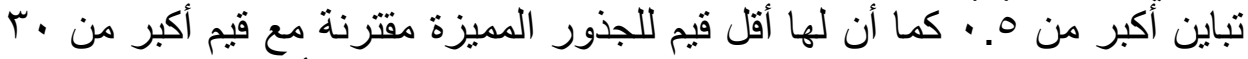

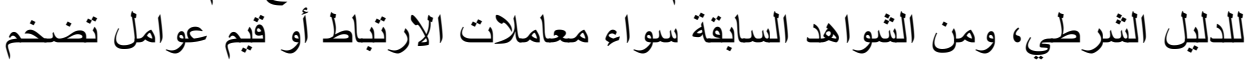
التباين، نستنتج أيضا أن المتغيرات التعدد الخطي. 
الجذور المميزة والدليل الثرطي ونسب تحلل التباين لمتغيرات السعر العالمي للقمح فضلا عن مجموعة متغيرات الأزمات الدولية

\begin{tabular}{|c|c|c|c|c|c|c|c|c|c|c|c|c|c|c|c|c|}
\hline Number & & $\begin{array}{c}\text { Conditisun } \\
\text { Inter }\end{array}$ & $\begin{array}{c}\text { Var } \\
\text { Prop XI }\end{array}$ & $\begin{array}{c}\text { Var } \\
\text { Prep } x 2\end{array}$ & $\begin{array}{c}V_{m e} \\
\text { Prop } x 3\end{array}$ & $\begin{array}{c}\text { Var } \\
\text { Prop } X 4\end{array}$ & $\begin{array}{c}\text { Var } \\
\text { Prop XS }\end{array}$ & $\begin{array}{c}\text { Var } \\
\text { Prap X6 }\end{array}$ & $\begin{array}{c}\text { Var } \\
\text { Prop X7 }\end{array}$ & $\begin{array}{c}\text { Yar } \\
\text { Prop XE }\end{array}$ & $\begin{array}{c}\text { Var } \\
\text { Prapi Xo }\end{array}$ & $\begin{array}{l}\text { Yar } \\
\text { Prop } \\
\text { XI6 }\end{array}$ & $\begin{array}{l}\text { Yar } \\
\text { Prap } \\
\text { XII }\end{array}$ & $\begin{array}{l}\text { Yar } \\
\text { rop } \\
\mathrm{X} 12\end{array}$ & $\begin{array}{l}\mathrm{V}_{\mathrm{ker}} \\
\mathrm{P}_{\mathrm{v}} \\
\mathrm{X} 13\end{array}$ & $\begin{array}{l}\text { Var } \\
\text { Prey } \\
\text { X14 }\end{array}$ \\
\hline 1 & & 1 & cotoz & 0601 & 0 & 0 & B & 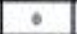 & & 0 & & $\cdot$ & .0913 & $0,60 \mathrm{I}$ & 0.0013 & 9.9604 \\
\hline 2 & เ.มระเ1 & 24713 & 0.960 & 0 & 0 & 0 & 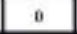 & 6 & 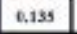 & & & $\bullet$ & 00007 & 80246 & 0.60097 & $4906 ?$ \\
\hline 3 & 0.30245 & 3.76281 & Q.2605 & 0 & 0 & 0 & a & t & anes & 0 & A & - & amst & 0015 & 0.0579 & 9.1097 \\
\hline 4 & n.seass & 4.34051 & 9.962 & 0 & 0.001 & 0 & 0 & 6 & 80001 & D.0015 & 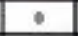 & $\bullet$ & 0.5nT9 & 0.0003 & 6.601 & o.acts \\
\hline 5 & 0.57361 & 4.45157 & 0.9015 & 4.9061 & 0 & 0 & 0 & 0 & 00186 & 0.0052 & A & $\cdot$ & 0.034 & 0.103 & 0.050 & .9604 \\
\hline 6 & 0.2854 & 4.36018 & 0.0655 & 9.00012 & 0 & 9 & 0 & e & 00501 & a.063 & 0 & $\bullet$ & oosss & 02838 & 6.25s9 & 9,9583 \\
\hline 7 & D.170u2 & 8.17654 & 0.0126 & 6.0031 & Q.6025 & 0 & 0 & 6 & 0.0218 & 6.0052 & 0 & $\bullet$ & $0.072 ?$ & 02465 & 6.2733 & A.978 \\
\hline 8 & a.ens & 13.2959 & 0.1051 & 9.9166 & 0.6057 & 0 & 0 & 0 & 0.0114 & 6.102 & $\circ$ & $\bullet$ & anss & (10331 & 0.0528 & Gatss \\
\hline$y$ & Mosies & 19.1328 & 0.1563 & 0.009 & 0.0994 & 0.901 & 4.0014 & 0.9514 & 0.958 & avist & 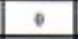 & 00301 & 0.1141 & 000367 & 0.0013 & อ.งาน \\
\hline 16 & D. .11349 & 29.1249 & 0.613 & 6.19 & to.00s & 0.0006 & (⿻,80)1 & anov & $0003 s$ & 80034 & 0 & $\bullet$ & a.2u19 & 0.035 & 0.0133 & 4.414? \\
\hline 11 & A.10555 & 44.6636 & 0.0643 & 0.179 & 0.0343 & 0.0045 & 0,0257 & a.2a25s & 0.0301 & 0.0012 & 0 & oones & Q02274 & 0.045s & augis & 0.9025 \\
\hline 12 & a.so293 & 62.2788 & 0.9659 & 0.451 & 0.6244 & 6.0096 & 0,0025 & 0,9613 & 0.0364 & 0.4 & $\theta$ & 0.012 & 0 & o.sous & B.037 & a.9.57 \\
\hline I3 & Q001478 & 154251 & 8.0032 & 0.002 & 8.6051 & 0.2695 & 6.2545 & 0.261 & $0.120 \mathrm{~s}$ & DJISs & 00007 & a015s & 0.0619 & 0.010 & 0.0145 & 6,0143 \\
\hline 14 & Q9ve19 & 243.456 & 0.9693 & 0.0043 & 0.0019 & 0.spiss & 4,7131 & 0.7671 & 0.5552 & 0.3774 & 0.014 & 0.152 & 2.0603 & $0.4 \omega$ & 0.1513 & MG45 \\
\hline 15 & 3.ANE.-16 & 1828 & 0,0182 & 0.125 & 0,178 & 0.1199 & 6.0031 & a.9045 & 0.042 & 0.0216 & 0.9972 & 0.9963 & aoss & 0.9965 & 0.0487 & 6.513 \\
\hline
\end{tabular}

و من أجل معالجة هذه المشكلة فقد تم اللجوء إلى استخدام أسلوب التحليل العاملي

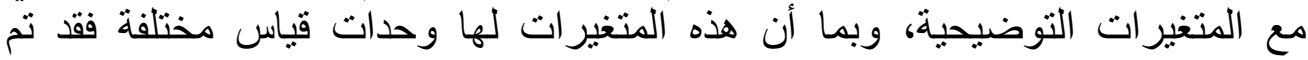

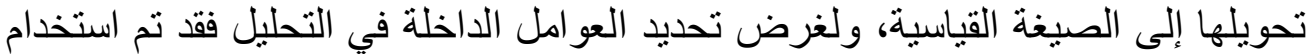

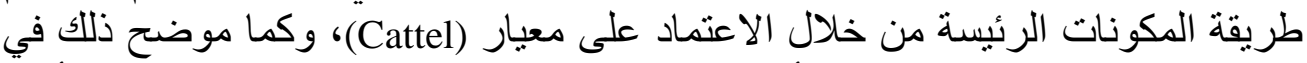

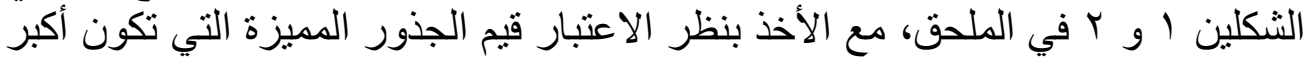

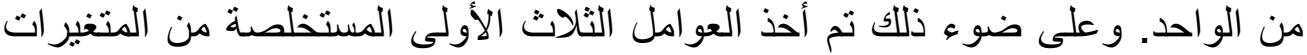

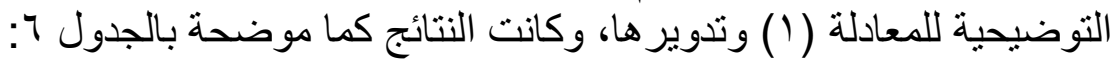

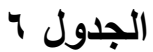

تحميلات العوامل المدورة لمصفوفة الارتباط لمتفيرات السعر العالمي للقمح باستبعاد متغيرات الأزمات الدولية العيرات 


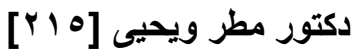

\begin{tabular}{|c|c|c|c|c|}
\hline Variable & Factor1 & Factor2 & Factor3 & Communality \\
\hline $\mathrm{X}_{1}$ & 0.443 & -0.846 & -0.089 & 0.919 \\
\hline $\mathrm{X}_{2}$ & 0.053 & -0.957 & -0.024 & 0.920 \\
\hline $\mathrm{X}_{3}$ & 0.601 & -0.552 & -0.138 & 0.685 \\
\hline $\mathrm{X}_{4}$ & 0.928 & -0.275 & -0.100 & 0.948 \\
\hline $\mathrm{X}_{5}$ & 0.941 & -0.269 & 0.024 & 0.959 \\
\hline $\mathrm{X}_{6}$ & 0.939 & -0.279 & -0.003 & 0.959 \\
\hline $\mathrm{X}_{7}$ & 0.011 & 0.089 & 0.994 & 0.996 \\
\hline $\mathrm{X}_{8}$ & 0.950 & -0.027 & 0.013 & 0.903 \\
\hline $\mathrm{X}_{9}$ & 0.971 & -0.213 & 0.023 & 0.988 \\
\hline $\mathrm{X}_{10}$ & 0.967 & -0.227 & 0.023 & 0.987 \\
\hline Variance & 5.9688 & 2.2685 & 1.0278 & 9.2652 \\
\hline$\%$ Var & 0.597 & 0.227 & 0.103 & 0.927 \\
\hline
\end{tabular}

من الجدول 7 نلاحظ أن العامل الأول وبعد التدوير فسر (59.7\%) من التباين

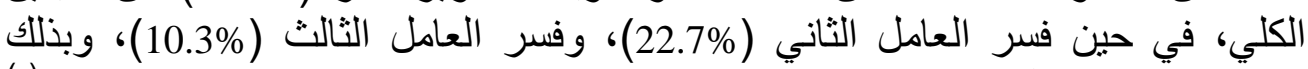

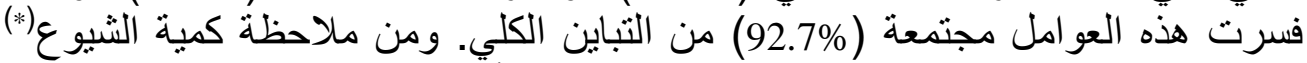
لمكن القول إن المتغير (Communality)

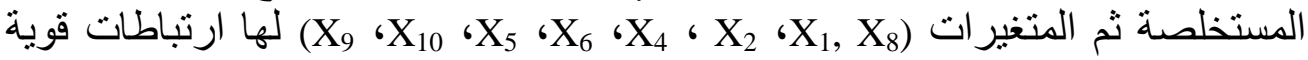
وحسب الترتيب، في حين يظهر المتغير (X3) بارتباط جزئي مع العوامل الثلاثة المستخلصة. - ابت

تحديد العوامل المؤثرة في السعر العالمي للقمح من دون متغيرات الأزمات

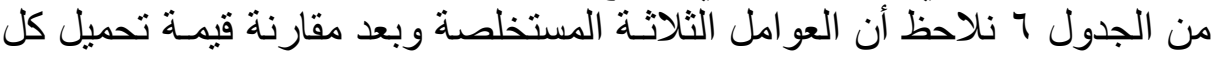

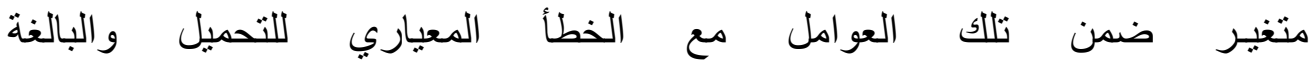
للعوامل الثلاثة على التوالي، نلاحظ من

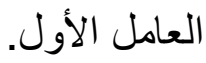

(*) هي عبارة عن قيم موجبة تقع بين الصفر و الواحد الصحيح إذ تظهر مدى التداخل بين المتغيرات

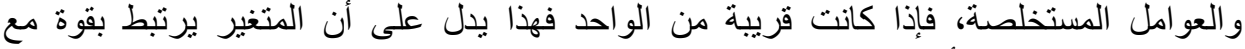

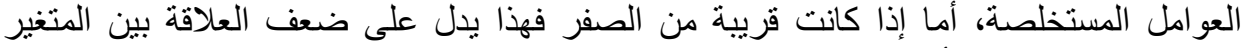

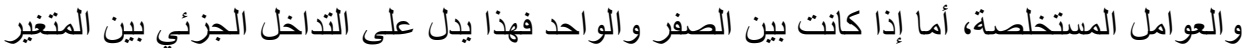

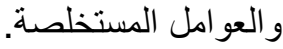


أن المتغير ات المتشبعة عليه كانت (X) X

يلاحظ أن المتغير ات

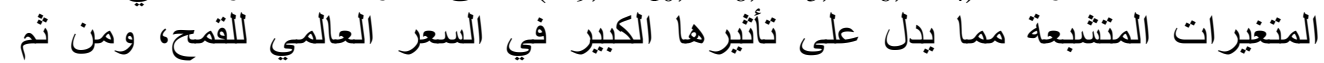

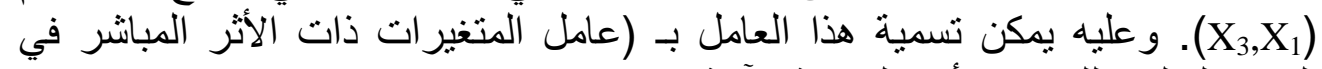
السعر العالمي للقمح) ويأخذ الصيغة الآنية:

$$
\begin{gathered}
\mathbf{F}_{1}=\frac{1}{5.9688}\left(0.443 * X_{1}+0.601 * X_{3}+0.928 * X_{4}+0.941 * X_{5}+0.939 * X_{6}\right. \\
\left.+0.950 * X_{8}+0.971 * X_{9}+0.967 * X_{10}\right)
\end{gathered}
$$

وفرز العامل الثاني المتغير ات المتشبعة (X, X

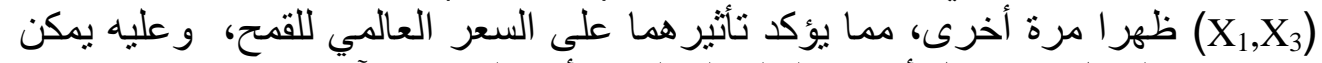

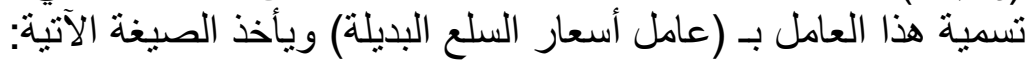

$\mathbf{F}_{2}=\frac{1}{2.2685}\left(-0.846 * X_{1}-0.957 * X_{2}-0.552 * X_{3}\right)$

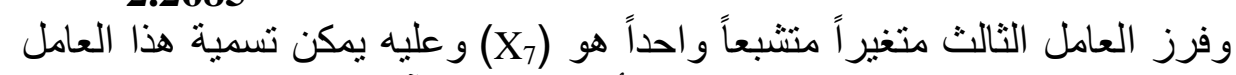
بـ (عامل المخزون العالمي لمحصول القمح) ويأخذ الصيغة الصئة الآتية: $F_{3}=\frac{1}{1.0278}\left(0.994 * X_{7}\right)$

تقدير معادلة السعر العالمي للقمح اعتمادا على العوامل الثثلاثة المستخلصة

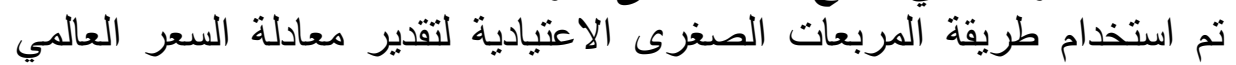
للقمح، وذلك من خلال انحدار السعر العالمي للقهح على العوامل المل المستخلصة

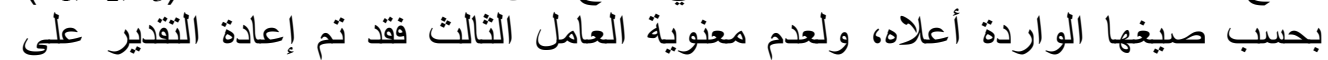
العاملين

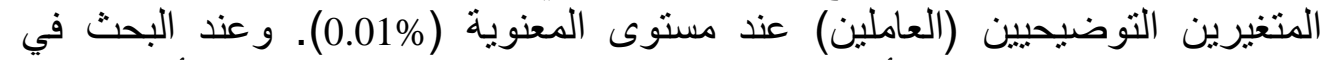

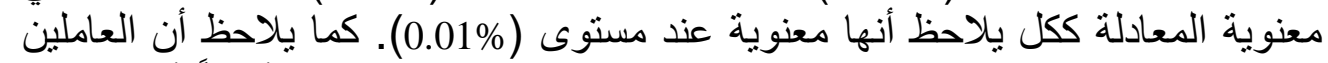

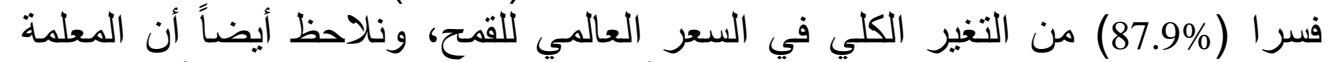

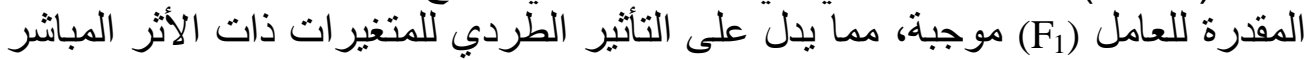

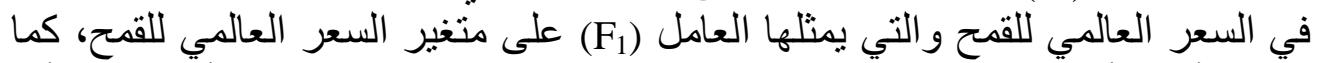

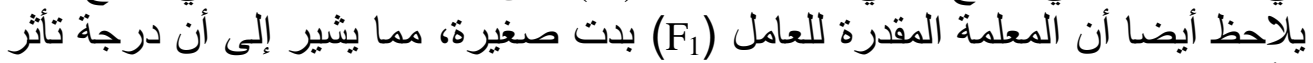

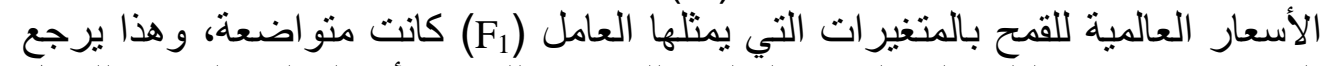

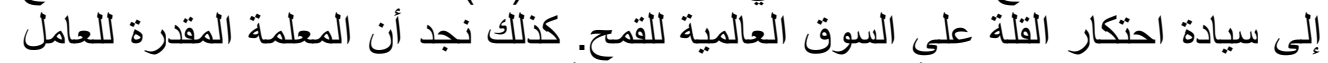

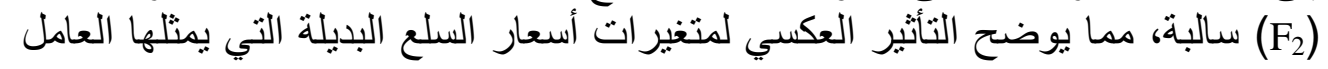


[YIV] دكتور مطر ويحيى

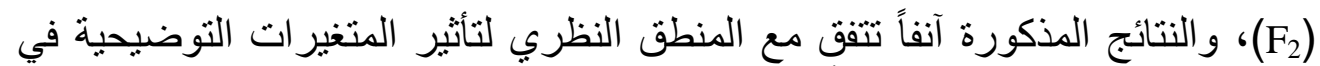

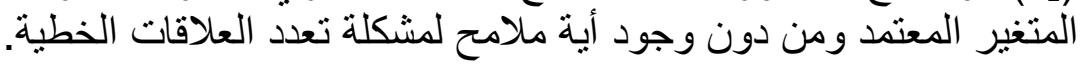

\begin{tabular}{|c|c|c|c|c|c|}
\hline \multicolumn{6}{|c|}{ 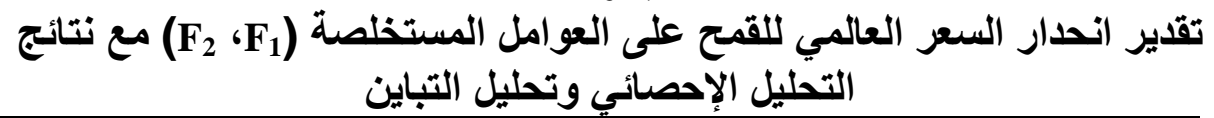 } \\
\hline Predictor & Coef & \multicolumn{2}{|c|}{ SE Coef } & $\mathbf{T}$ & $\mathbf{P}$ \\
\hline Constant & -32.90 & \multicolumn{2}{|c|}{10.07} & -3.27 & 0.002 \\
\hline $\mathrm{F} 1$ & 0.0019352 & \multicolumn{2}{|c|}{0.0003324} & 5.82 & 0.000 \\
\hline F2 & -0.092313 & \multirow{2}{*}{\multicolumn{2}{|c|}{\begin{tabular}{c|}
0.007309 \\
$R-S q($ adj $)=87.3 \%$
\end{tabular}}} & -12.63 & 0.000 \\
\hline $\mathrm{S}=15.32$ & $\mathrm{R}-\mathrm{Sq}=87$ & & & & \\
\hline \multicolumn{6}{|c|}{ Analysis of Variance } \\
\hline Source & $\overline{\text { DF }}$ & SS & MS & $\mathbf{F}$ & $\mathbf{P}$ \\
\hline Regression & 2 & 66664 & 33332 & 142.10 & 0.000 \\
\hline Residual Erro & 39 & 9148 & 235 & & \\
\hline Total & 41 & 75812 & & & \\
\hline
\end{tabular}

تحديد العوامل المؤثرة في السعر العالمي للقمح بوجود متغيرات الأزمات الدولية

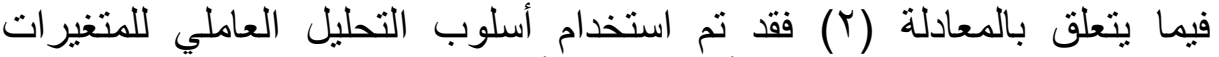

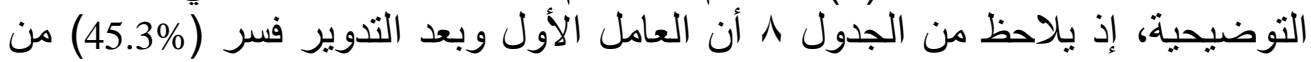

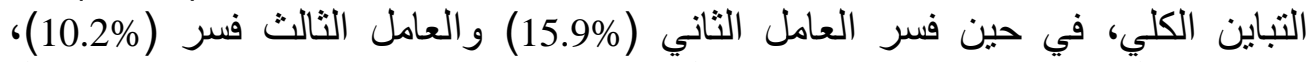

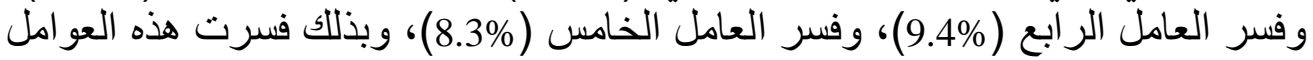
مجتمعة (89.1\%) من التباين الكلي، ومن ملاحظة كمية الثيوع الثيو يمكن القول إن المنتغير

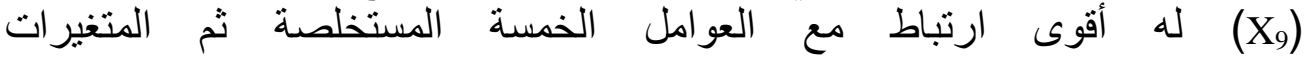
لها ارتباطات قوية وحسب الترتيب، في حين تظهر (X) المتغير ات (X) بارتباطات جزئية مع العو امل الخمسة المستخلصة.

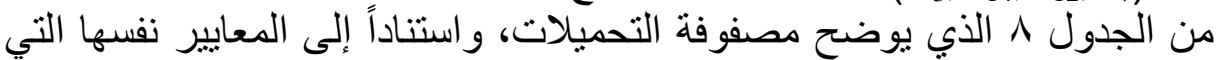

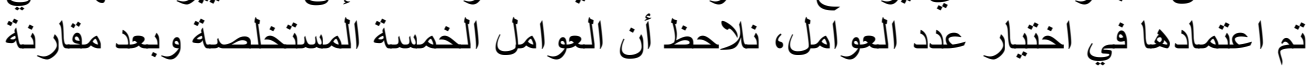

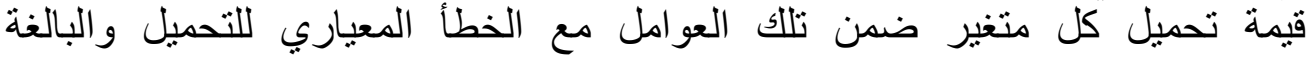
$\left(\mathrm{S}_{1}=0.1986, \mathrm{~S}_{2}=0.3352, \mathrm{~S}_{3}=0.4182, \mathrm{~S}_{4}=0.4347, \mathrm{~S}_{5}=0.4625\right)$

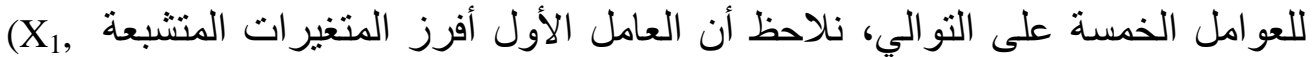

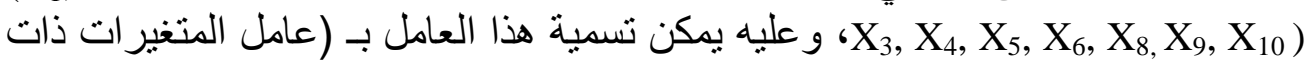


الأثر المباثر على السعر العالمي للقمح) و هي المتغير ات نفسها التي تشبعت على العامل الأول لمتغير ات المعادلة (1) ويأُخذ العامل الأول هنا الصيغة الآتية:

$$
\begin{gathered}
\mathbf{F}_{1}^{*}=\frac{1}{6.3352}\left(0.527 * X_{1}+0.667 * X_{3}+0.942 * X_{4}+0.96 * X_{5}+0.959 * X_{6}\right. \\
+0.952 * X_{8}+0.984 * X_{9}+0.981 * X_{10}
\end{gathered}
$$

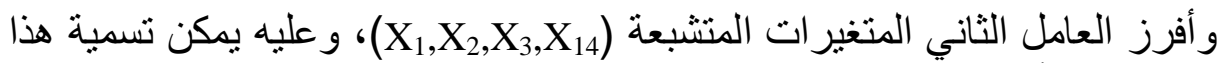

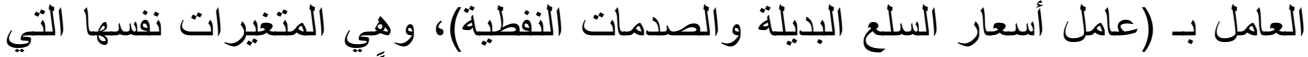

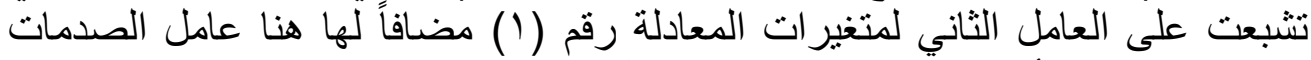
النفطية (X14)، ويأخذ العامل الثاني الصيغة الآتية: $F_{2}^{*}=\frac{1}{2.224}\left(-0.761 * X_{1}-0.944 * X_{2}-0.419 * X_{3}-0.614 * X_{14}\right)$

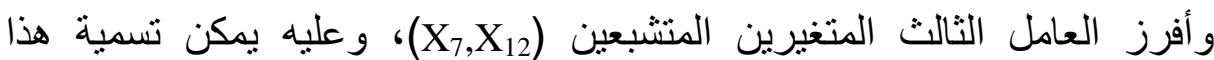
العامل بـ (عامل التغير في المخزون العالمي والجفاف) ويأخذ الصيغة الآتية:

$$
F_{3}^{*}=\frac{1}{1.4291}\left(0.816 * X_{7}+0.844 * X_{12}\right)
$$

و أفرز العامل الرابع تثبع المتغيرين (X)

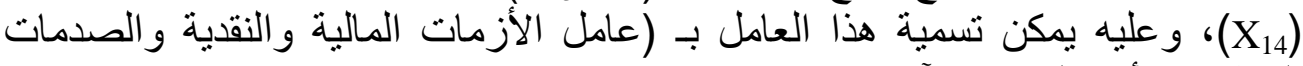
النفية) ويأخذ الصيغة الآتية: $F_{4}^{*}=\frac{1}{1.3228}\left(-0.916 * X_{13}+0.644 * X_{14}\right)$

وأفرز العامل الخامس تتبع المتغير (XX))، وعليه يمكن تسمية هذا العامل بـ (عامل الحروب) ويأخذ الصيغة الآتية:

$$
F_{5}^{*}=\frac{1}{1.1686}\left(-0.973 * X_{11}\right)
$$




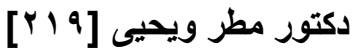

تحميلات العوامل المدورة لمصفوفة الارتباط لمتغيرات السعر العالمي للقمح فضلا عن

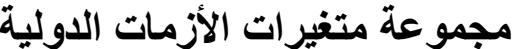

\begin{tabular}{|c|c|c|c|c|c|c|}
\hline Variable & Factor1 & Factor2 & Factor3 & Factor4 & Factor5 & Communality \\
\hline $\mathrm{X}_{1}$ & 0.527 & -0.761 & -0.061 & -0.112 & 0.130 & 0.891 \\
\hline $\mathrm{X}_{2}$ & 0.151 & -0.944 & -0.027 & -0.029 & -0.042 & 0.918 \\
\hline $\mathrm{X}_{3}$ & 0.667 & -0.419 & -0.174 & 0.076 & 0.374 & 0.797 \\
\hline $\mathrm{X}_{4}$ & 0.942 & -0.198 & -0.075 & -0.137 & -0.068 & 0.956 \\
\hline $\mathrm{X}_{5}$ & 0.960 & -0.183 & 0.044 & -0.068 & -0.062 & 0.967 \\
\hline $\mathrm{X}_{6}$ & 0.959 & -0.193 & 0.024 & -0.064 & -0.052 & 0.964 \\
\hline $\mathrm{X}_{7}$ & 0.030 & 0.169 & 0.816 & 0.027 & -0.112 & 0.709 \\
\hline $\mathrm{X}_{8}$ & 0.952 & 0.068 & -0.043 & 0.115 & 0.082 & 0.932 \\
\hline $\mathrm{X}_{9}$ & 0.984 & -0.121 & -0.005 & -0.065 & -0.031 & 0.989 \\
\hline $\mathrm{X}_{10}$ & 0.981 & -0.139 & 0.001 & -0.064 & -0.035 & 0.987 \\
\hline $\mathrm{X}_{11}$ & 0.064 & 0.025 & -0.060 & 0.016 & -0.973 & 0.955 \\
\hline $\mathrm{X}_{12}$ & -0.068 & -0.090 & 0.844 & -0.009 & 0.166 & 0.752 \\
\hline $\mathrm{X}_{13}$ & 0.109 & -0.121 & -0.036 & -0.916 & 0.030 & 0.867 \\
\hline $\mathrm{X}_{14}$ & 0.014 & -0.614 & -0.042 & 0.644 & 0.051 & 0.796 \\
\hline Variance & 6.3352 & 2.2240 & 1.4291 & 1.3228 & 1.1686 & 12.4797 \\
\hline$\%$ Var & 0.453 & 0.159 & 0.102 & 0.094 & 0.083 & 0.891 \\
\hline
\end{tabular}

\section{تقدير معادلة السعر العالمي للقمح اعتماداً على العوامل الخمسة المستخلصة}

تم استخدام طريقة المربعات الصغرى الاعتيادية لتقدير معادلة السعر العاديالة العالمي

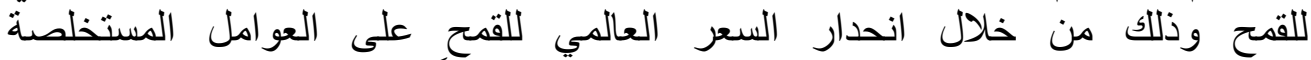

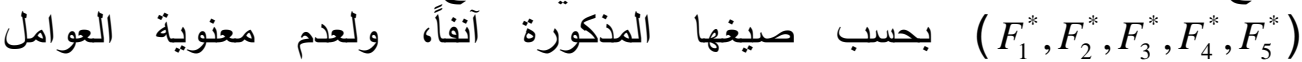
فقد تمت إعادة التقدير على العاملين ( $\left.F_{3}^{*}, F_{4}^{*}, F_{5}^{*}\right)$

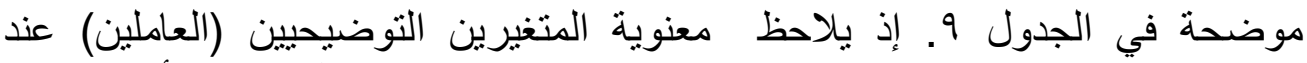
مستوى المعنوية (0.01\%) .و عند البحث في معنوية المعادلة ككل نلاحظ أنها معنوية عند مستوى (0.01\%). كما يلاحظ أن العاملين فسرا (87.5\%) من التغنير التغير الكلي في

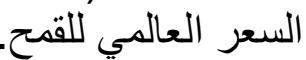

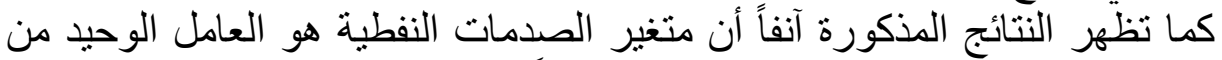

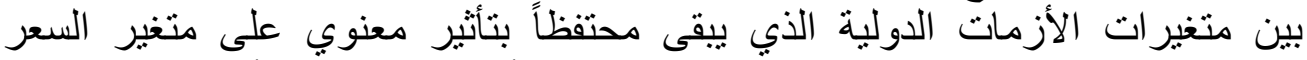

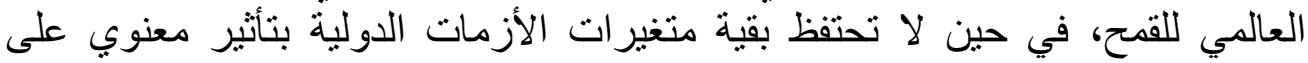

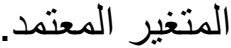

\section{الجدول 9}




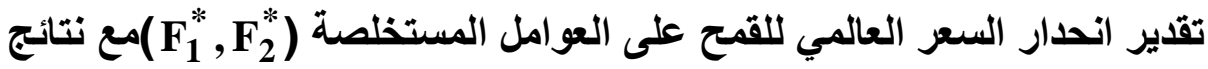
التحليل الإحصائي وتحليل التباين

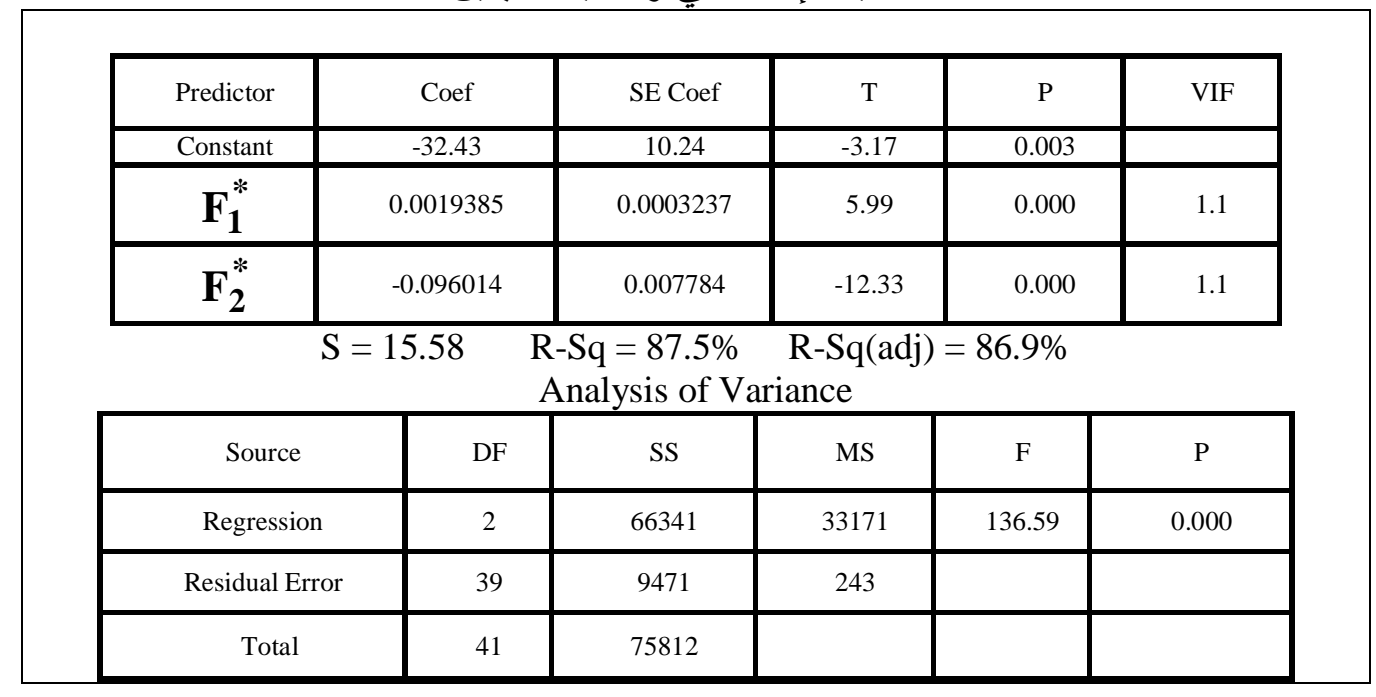

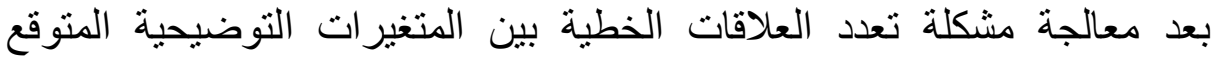

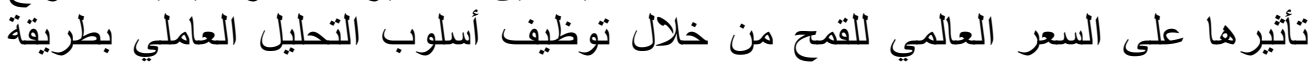

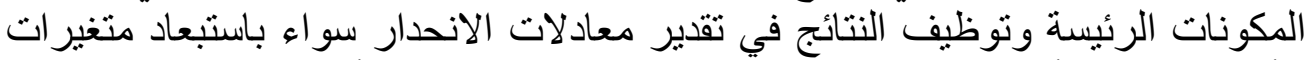

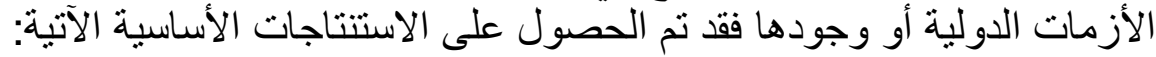

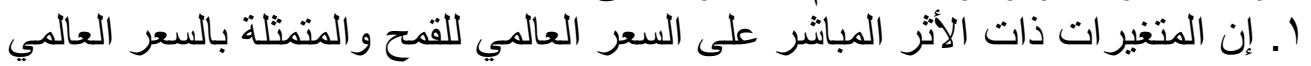

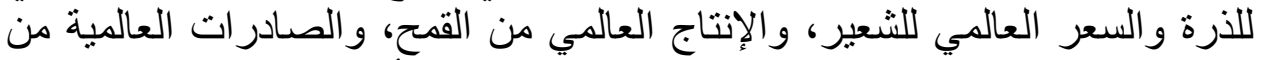

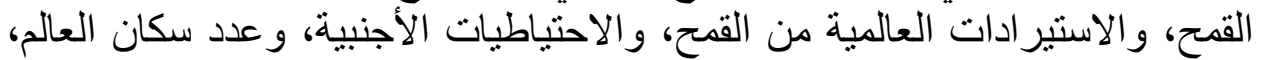

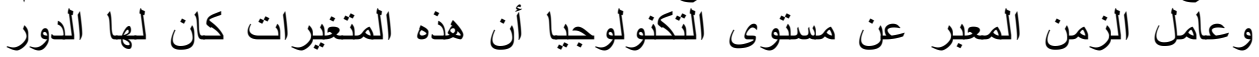

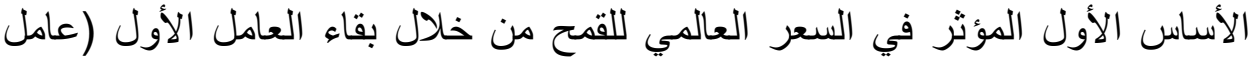

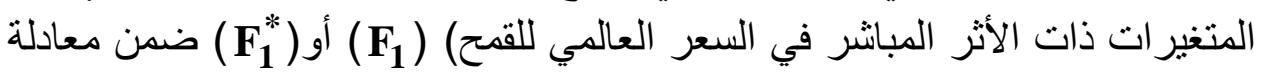

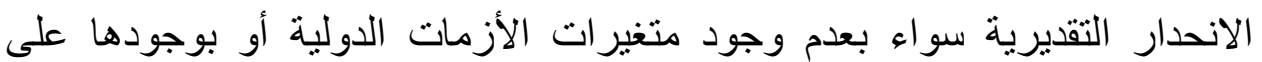

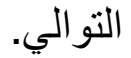

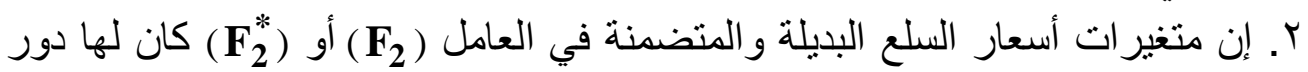

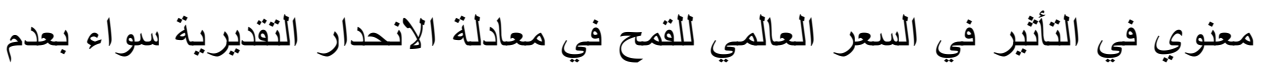

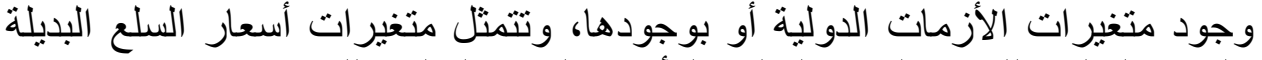
بالسعر العالمي للذرة والسعر العالمي للأرز والسعرد السعر العالمي للشعير. 


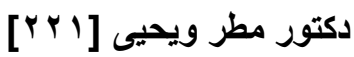

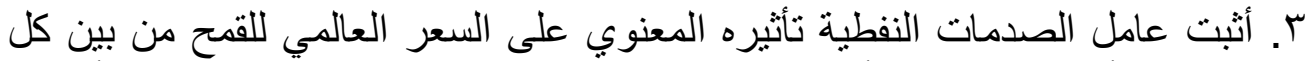

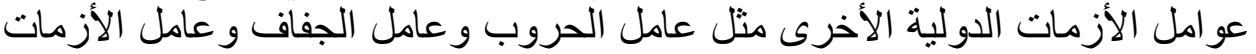

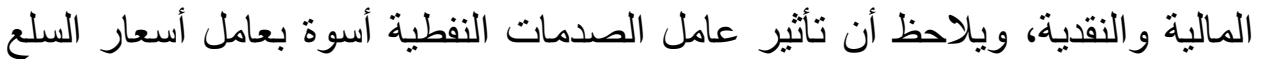

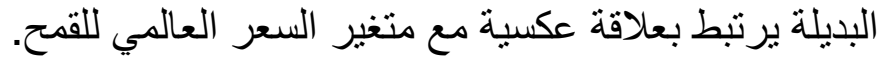

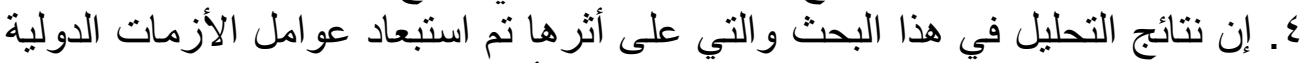

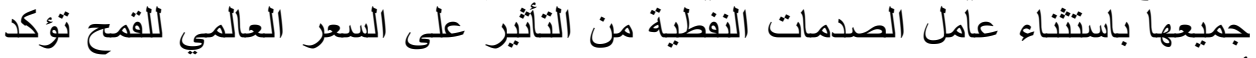

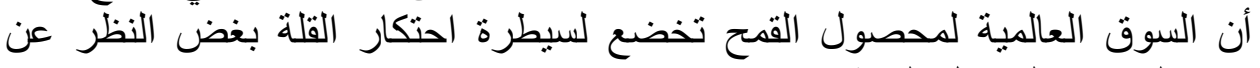
قوى السوق و العو امل المؤثرة فيها.

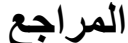 \\ أولاًا-المراجع باللغة العربية}

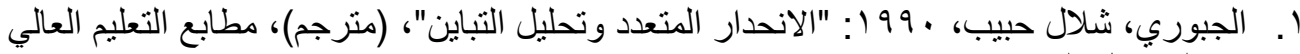

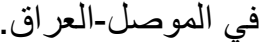

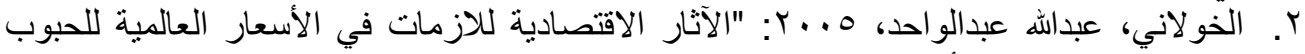

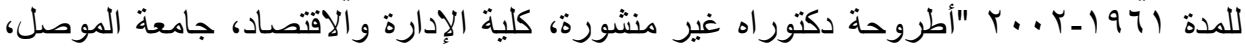
r. سالفاتور، دومينيك، ب919 1: "الإحصاء والاقتصاد القياسي"، ملخصات شوم، دار ماكجروهيل

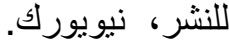
؛. و وارطان، هاسميك انترانيك، 1919 1: "تعدد العلاقات الخطية في نموذج الانحدار"، رسالة ماجستير غير منشورة، كلية الإدارة والاقتصاد، جامعة الموصل. 


\section{ثانياً. المراجع باللغة الاجنية}

1. Brown,A.T.(2006);"Confirmatory Factor Analysis for Applied Research",The Guilford Press ،New York.

2. Gujarati,D.N. (1988); "Basic Econometrics" , McGraw - Hill Book Company, New York.

3. Intrilligator, M.D. (1996);"Econometrics Models, Techniques and Applications", Prentice Hall.

4. Manly,B.F.J.(2004);"Multivariate Statistical Methods", $3^{\text {rd }}$ ed. Chapman \& Hall, CRC Press Company, Washington D.C.

5. Ronald P.C \& Jeffrey K.S.(2006) "Applied Statistics and the SAS Programing Language" , $5^{\text {th }}$ ed. ,Pearson Prentice Hall, Pearson Education, Inc. USA. 